\title{
Induction of human IL-10-producing neutrophils by LPS-stimulated Treg cells and IL-10
}

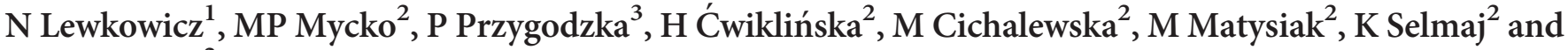 \\ P Lewkowicz ${ }^{2}$
}

Recent evidence has revealed an unsuspected suppressive role played by neutrophils during microbial infections. An especially intriguing aspect of this role is the ability of neutrophils to produce interleukin (IL)-10 following interaction with lipopolysaccharide (LPS)-stimulated regulatory $\mathrm{T}$ (Treg) cells. The present study demonstrates that generation of IL-10 in neutrophils induced by LPS-stimulated Treg cells required direct cell-cell contact. This effect was dependent on the binding of CD11b and intercellular adhesion molecule 1. Neither stimulation of neutrophils with LPS nor their culture with unstimulated Treg cells, CD3/CD28 monoclonal antibodies-stimulated Treg cells, or T conventional cells affected intracellular IL-10 expression. IL-10-positive neutrophils were also induced by exogenous IL-10, providing an example of a positive feedback loop. Both LPS-stimulated Treg cells and exogenous IL-10 exclusively promoted posttranslational modifications of histones, $\mathrm{H} 3 \mathrm{~K} 4 \mathrm{me} 3$ and $\mathrm{H} 3 \mathrm{Ac}$ Lys4, that activate $\mathrm{IL}-10$ genomic locus in neutrophils, while the promoter of IL-10 gene was inactive in resting, LPS-stimulated neutrophils, following blocking of direct interaction with LPS-stimulated Treg cells or in LPS-preactivated neutrophils incubated with LPS-stimulated Treg cells. We additionally confirmed the presence of IL-10-producing neutrophils in vivo in patients with periodontal abscess induced by Gram-negative bacteria, as opposed to neutrophils isolated from the site of aseptic inflammation in patients with neuromyelitis optica.

\section{INTRODUCTION}

Neutrophils are the first cells that migrate to the site of infection to eliminate microorganisms. They also recruit other innate cells, including monocytes and dendritic cells through the release of chemokines and antimicrobial peptides. ${ }^{1}$ Recently, neutrophils have been found to have a previously unsuspected suppressive role during acute and chronic microbial infection. Several murine studies describe the recruitment process of IL-10-producing neutrophils during bacterial, fungal, or parasitic infection. $^{2-7}$ These suppressive neutrophils control the inflammatory response of dendritic cells, monocytes, macrophages, and $\mathrm{T}$ helper type 1 cells. ${ }^{2,5,7}$ In contrast to murine neutrophils, there is no consensus as to whether human neutrophils are capable of IL-10 production. Although the results of two recent studies show that IL-10-producing human neutrophil induction takes place in response to lipopolysaccharide (LPS) and serum amyloid A, which suppress the antigen-specific proliferation of $\mathrm{CD} 8{ }^{+} \mathrm{T}$ cells, these findings have not been confirmed in other studies. ${ }^{8-10}$ Both reports reveal significant contradictions that might be related to the different purification protocols and experimental settings used. The lack of ability of human neutrophils to produce IL-10 was explained by inactive chromatin configuration at the $I L-10$ locus, contrary to human monocytes or murine neutrophils. ${ }^{11}$ More recently, IL-10 production was demonstrated in human neutrophils in response to Paracoccidioides brasiliensis. ${ }^{12}$ The present study proposes an alternative mechanism of IL-10 induction in human neutrophils involving LPS-stimulated Treg cells.

Our earlier articles describe a mechanism based on the suppression of neutrophils by LPS- or CD3/CD28 monoclonal antibodies (mAbs)-activated Treg cells and exclusive role of LPS-stimulated Treg cells that promote the generation of suppressive neutrophils, which produce IL-10, transforming

${ }^{1}$ Department of Periodontology and Oral Mucosal Diseases, Medical University of Lodz, Lodz, Poland. ${ }^{2}$ Department of Neurology, Laboratory of Neuroimmunology, Medical University of Lodz, Lodz, Poland and ${ }^{3}$ Institute of Medical Biology, Polish Academy of Sciences, Lodz, Poland. Correspondence: P Lewkowicz (przemyslaw.lewkowicz@umed.lodz.pl) 
growth factor- $\beta 1$, and express indoleamine-2,3-dioxygenase, suppressor of cytokine signaling 3 , and heme oxygenase-1. ${ }^{13,14}$ These data uncover neutrophils as important negative regulators of the immune response during LPS-mediated inflammation. However, the mechanism responsible for this exclusive function of LPS-activated Treg cells remains elusive. Our data were in line with the study reporting that the intravenous administration of LPS in humans was followed by the appearance of a specific subset of neutrophils, which inhibited T-cell proliferation through CD11b. ${ }^{15}$ In addition, a significant increase of $\mathrm{CD} 11 \mathrm{~b}$ expression was observed on neutrophils cocultured with Treg cells. ${ }^{13}$ On the basis of these results, it can be hypothesized that CD11b might have a crucial role in neutrophil-Treg cell interactions, providing a signal that mediates the suppressive profile of the neutrophils.

In this study, we analyzed the mechanisms responsible for the exclusive function of LPS-activated Treg cells that contribute to the induction of IL-10-producing neutrophils. Our study showed that LPS-activated Treg cells significantly upregulate IL-10 production by neutrophils. This function of LPS-activated Treg cells was dependent on the direct cell-cell contact with neutrophils. Neither LPS stimulation of neutrophils nor their culture with unstimulated Treg cells, CD3/ CD28-stimulated Treg cells, or T conventional (Tconv) cells affected intracellular IL-10 level or the percentage of IL-10positive neutrophils. Moreover, a system of positive feedback was identified, insofar that IL-10 itself promoted IL-10 production by neutrophils, which explains how very few Treg cells are able to induce IL-10 secretion in a much larger population of neutrophils. We also showed that CD11bdependent interaction of neutrophils with LPS-stimulated Treg cells or incubation with IL-10 contributed to chromatin reorganization and posttranslational modification by methylation of $\mathrm{H} 3$ at $\mathrm{K} 4$ and acetylation of $\mathrm{H} 3$ histones at the $I L-10$ locus.

\section{RESULTS}

\section{Human neutrophils produce IL-10 after interaction with LPS-stimulated Treg cells}

No coherent set of results exist in the literature regarding IL-10 production by human neutrophils, as many different aspects might exert an influence on data collection. ${ }^{8-10,12,14}$ Our previous study has shown that LPS-activated Treg cells promote the generation of suppressive neutrophils that produce IL-10. ${ }^{14}$ To better understand the phenomenon of IL-10 production by human neutrophils and to confirm our previous findings, we have applied immunocytochemistry (ICC) as one of the technique of our investigations. This method allows neutrophils to be distinguished from possibly contaminating subsets of eosinophils or monocytes based on the morphological profile of these cells and thus dismiss doubts that were raised by some authors before. ${ }^{10}$ Additionally, immunochemical observations allowed to shorten the time of manipulation of cells that otherwise might influence the ability of neutrophils to produce IL-10.
The neutrophils were incubated alone or in the presence of LPS, unstimulated Treg cells, CD3/CD28-stimulated Treg cells, or LPS-stimulated Treg cells for 5-21 h, and then the intensity of intracellular IL-10 and the rates of IL-10-positive neutrophils were analyzed by immunocytochemistry. We also tested IL-10 protein production in cell culture supernatants and its mRNA expression in cell cocultures previously depleted of CD2positive cells.

It was found that untreated neutrophils produced very low but detectable amounts of IL-10 (Figure 1a-c). Incubation of freshly isolated neutrophils with LPS-activated Treg cells resulted in a 10-fold increase of the intensity of IL-10 fluorescence in ICC analysis, whereas unstimulated Tconv or Treg cells, CD3/CD28 mAb-activated Tconv or Treg cells, or LPS-stimulated Tconv cells had no effect on IL-10 production (Figure 1a). Treg cells remained negative for IL-10 under all the conditions used in this experiment (Figure 1a), which was in accordance with our previous enzyme-linked immunosorbent assay (ELISA), western blotting, and mRNA data. ${ }^{13}$ Preincubation of neutrophils with LPS for $15 \mathrm{~min}$ prior to addition of LPS-stimulated Treg cells completely inhibited IL-10 fluorescence (Figure 1a,c). To exclude the possibility of false positive results, control groups were created as necessary. Both the isotype control of IL-10 and secondary mAb control were negative, as the fluorescence signal was undetectable (Figure 1a).

Next, the intracellular localization of IL-10 in human neutrophils was confirmed by confocal microscopy with $z$-stack analysis (Figure 1b). Intracellular IL-10 was detected in about $7-9 \%$ of unstimulated CD15 ${ }^{+}$neutrophils, while about $65 \%$ of $\mathrm{CD} 15^{+}$neutrophils were positive for IL-10 when cultured with LPS-stimulated Treg cells for 5 or $21 \mathrm{~h}$ (Figure 1c). These findings were further confirmed by ELISA and real-time reverse transcriptase-PCR (RT-PCR; Figures 1d and $3 \mathrm{c}$ ). To rule out the possibility that LPS was responsible for the stimulation of IL-10 production in co-cultures with LPS-treated Treg cells, additional experiments were conducted on neutrophils incubated with $100 \mathrm{ng} \mathrm{ml}^{-1}$ ultrapure LPS. Inclusion of LPS surprisingly resulted in detection of decreased IL-10 levels (Figures 1a,c) measured as mRNA expression and release of IL-10 into extracellular space (Figure 3c).

\section{Polarization of neutrophils into IL-10-producing cells is dependent on the direct interaction with LPS-stimulated Treg cells via CD11b-ICAM-1}

The visualization of interactions between neutrophils and Treg cells revealed their close proximity (Figure 2). During a 7-hour incubation, a single LPS-stimulated Treg cell interacted with at least two neutrophils. As previous findings showed that the expression of CD11b on neutrophils incubated with unstimulated Treg cells was elevated, and Treg cells expressed high levels of intercellular adhesion molecule 1 (ICAM-1), ${ }^{16}$ CD11b on neutrophils and ICAM-1 on Treg cells were blocked in parallel to investigate whether CD11b/ICAM-1 synapse has a role in neutrophil-Treg cell interaction. Although physical contact of Treg cells and neutrophils was still visible, probably 
a
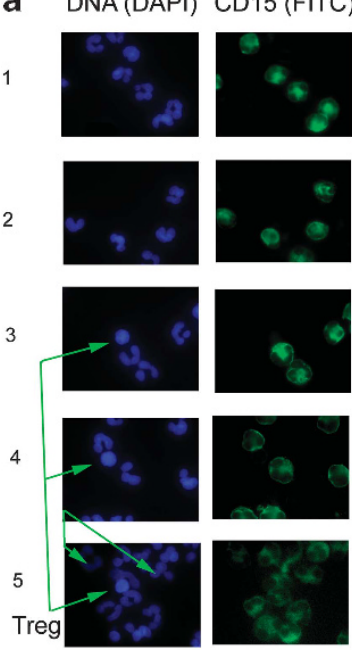

6
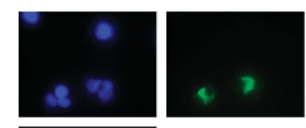

7
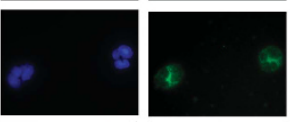

8
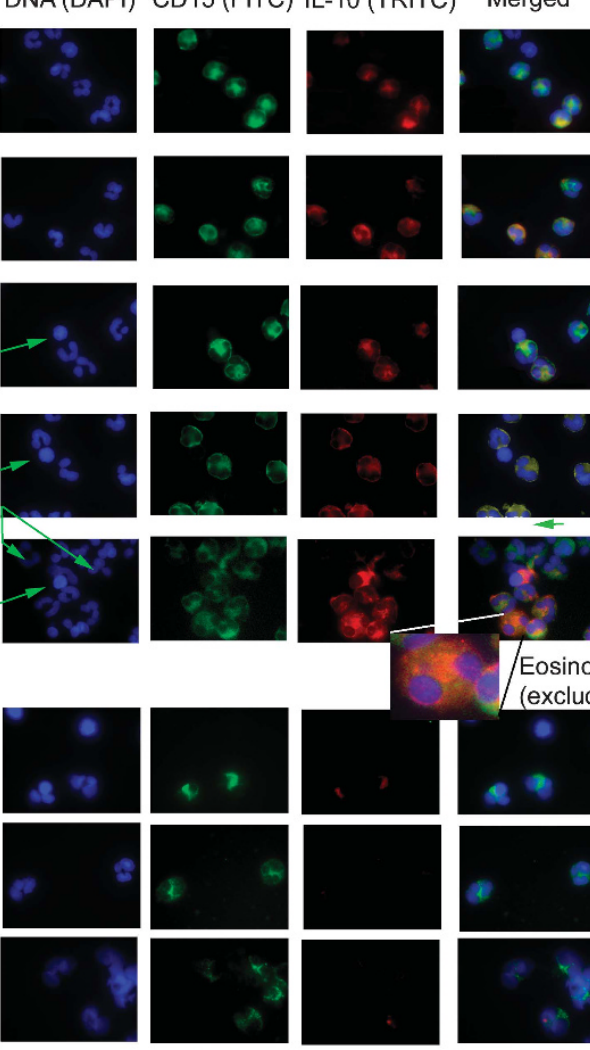

IL-10 segmentation
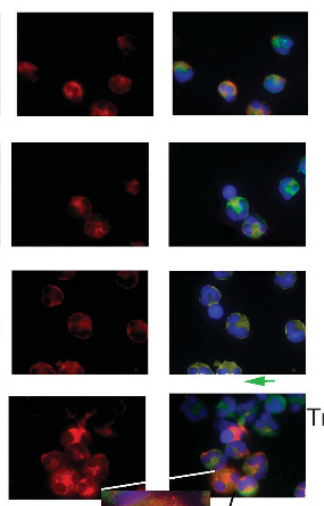

Eosinophil
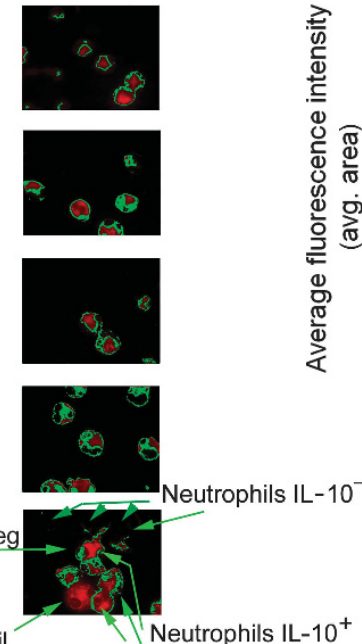

(excluded from analysis)
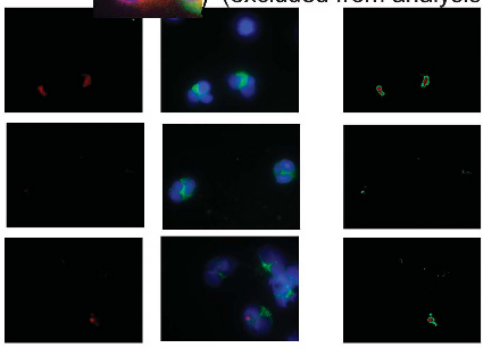

$30 \mu \mathrm{m}$

b

Neutrophils
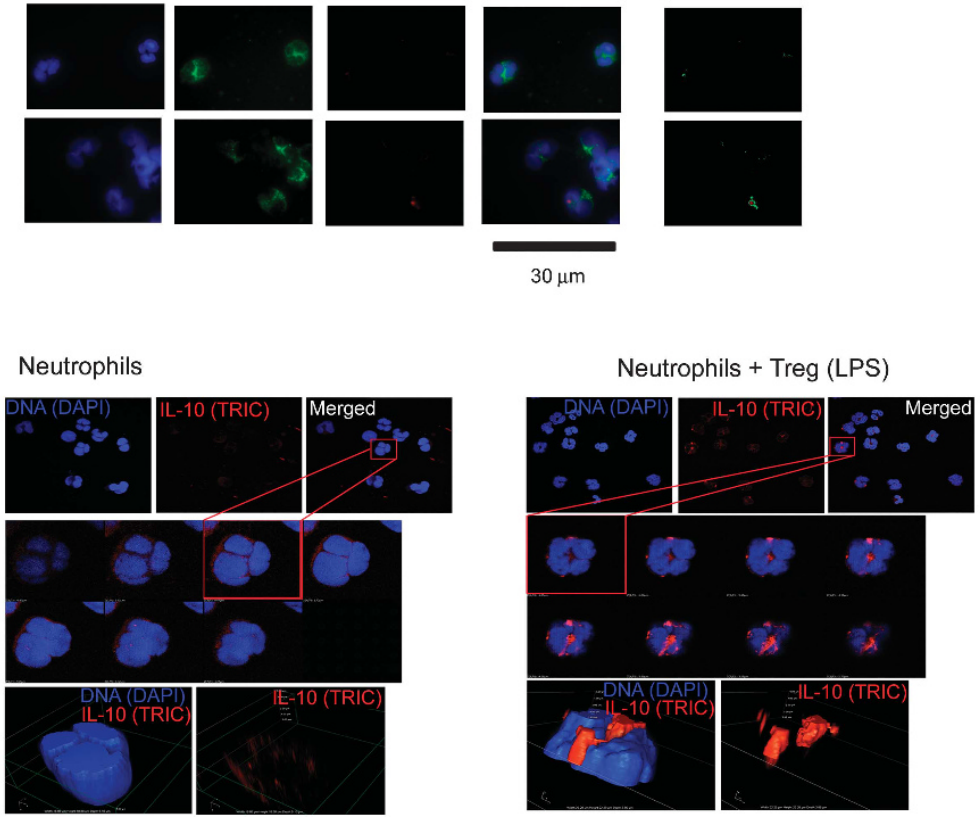
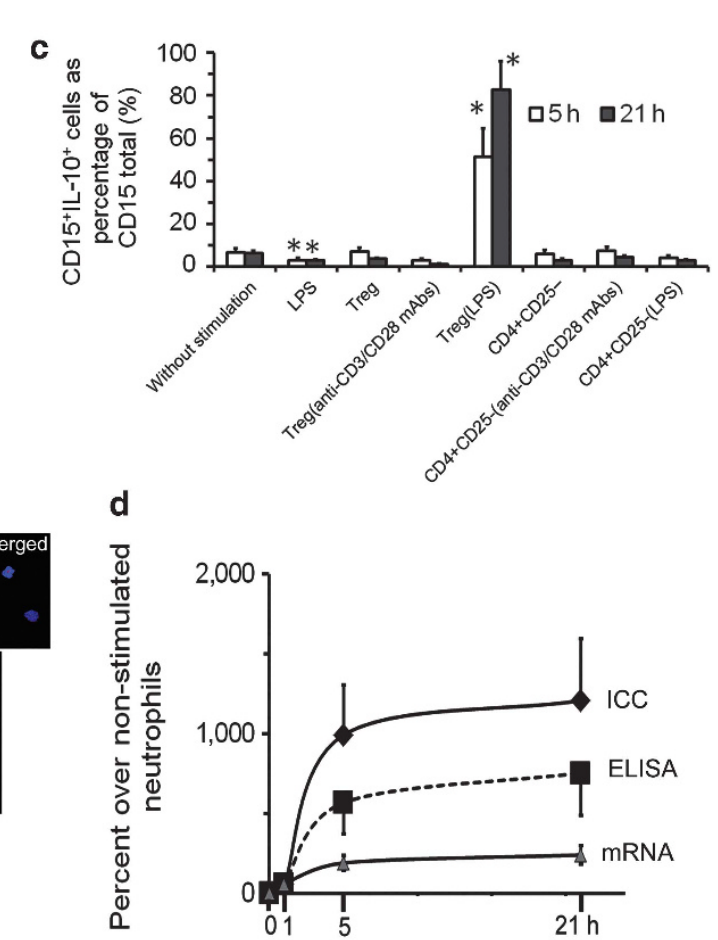

Figure 1 Lipopolysaccharide (LPS)-stimulated regulatory T (Treg) cells exclusively promote interleukin (IL)-10 production by human neutrophils. Preactivation of neutrophils with LPS completely abrogates this effect. (a) Neutrophils were cultured alone or in the presence of LPS, unstimulated, antiCD3/CD28 monoclonal antibodies (mAbs), or LPS-stimulated Treg cells. Fluorescence intensity was shown as the average fluorescence of the measured segments (sum of the fluorescence regions of all segments divided by the number of segment). ${ }^{15}$ The contaminating eosinophils and monocytes were identified based on the morphological shape of nuclei and excluded from further analysis: a " $U$ " or kidney-bean-shaped nucleus for the monocytes and a bi-lobed telephone receiver-like nucleus for the eosinophils. IL-10-negative Treg cells are indicated with arrows. (b) The intracellular localization of IL-10 in human neutrophils in the $z$-stack analysis. (c) The frequency of IL-10-producing CD15 ${ }^{+}$neutrophils cultured alone, in the presence of LPS, or together with preactivated Treg, conventional T cells at a ratio $50: 1$ for $5 \mathrm{~h}$. Data are presented as the percentage of total CD15 ${ }^{+}$cells. (d) Timecourse analysis of IL-10 production by neutrophils during incubation with LPS-stimulated Treg cells. Data are from four independent experiments. DAPI, 4,6-diamidino-2-phenylindole; ELISA, enzyme-linked immunosorbent assay; FITC, fluorescein isothiocyanate; ICC, immunocytochemistry; TRITC, tetramethylrhod-amine isothiocyanate. ${ }^{*} P<0.05$ compared to neutrophils without stimulation.

as a result of IL- 8 synthesis by Treg cells, ${ }^{17}$ blocking CD11b or ICAM-1 significantly affected IL-10 production by neutrophils. However, the induction of IL-10 in neutrophils by LPSstimulated Treg cells was found to be only partially inhibited in both situations, when CD11b or ICAM-1 were blocked
(Figure 3a-c). To definitively confirm the need of direct cell-cell contact in induction of IL-10-producing neutrophils, additional transwell experiments were performed. When we used the membrane that prevented direct cell-cell contact, the induction of IL-10 in neutrophils was completely inhibited 

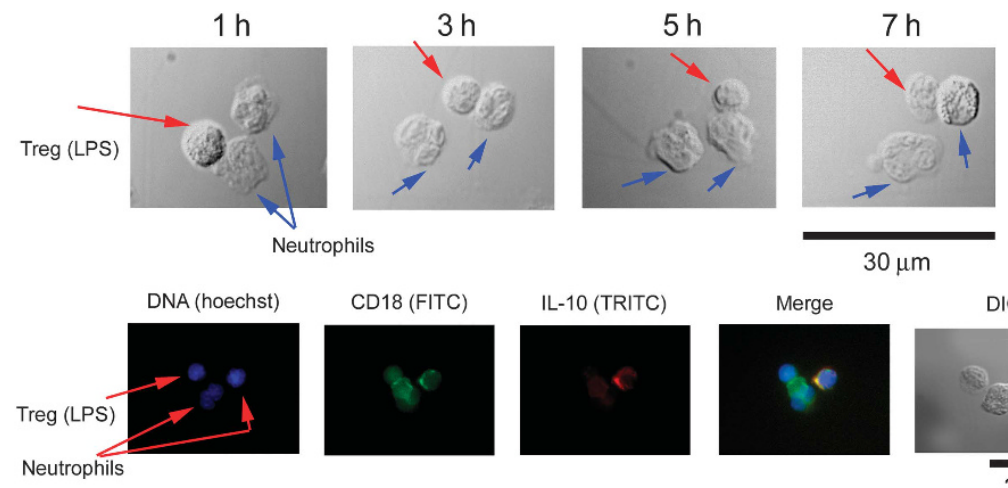

IL-10 (TRITC)
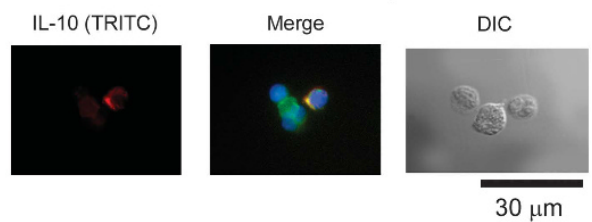

Figure 2 Direct visualization and time-lapse image of neutrophil regulatory T (Treg) cell interaction in 7-h culture. Single lipopolysaccharide (LPS)stimulated Treg cell interacted with two neutrophils (differential interference contrast images). After $7 \mathrm{~h}$, cells were fixed and stained with DNA-hoechst, CD18-FITC (fluorescein isothiocyanate), and interleukin (IL)-10-TRITC (tetramethylrhod-amine isothiocyanate) and florescence was visualized using a Zeiss Axiovert 200 inverse microscope. An example of one of the four independent experiments.

(Figure 3c). Changes in the surface expression of ICAM-1 on Treg cells and CD11b on neutrophils upon the conditions used in our experiments might be responsible for the final outcome regarding IL-10-producing neutrophil induction. Therefore, the expression of ICAM-1 by Tregs incubated with LPS or antiCD3/CD28 mAbs for $48 \mathrm{~h}$ was measured, and it was found that ICAM-1 density was upregulated in Treg cells by both stimulating factors, compared with non-stimulated Treg cells or activated Tconv cells (Figure 3d). Furthermore, CD11b expression was differentially regulated by Treg cells. CD11b was significantly upregulated in neutrophils incubated with non-stimulated or anti-CD3/CD28 mAbs, by contrast to incubation with LPS-stimulated Treg cells, where downregulation of $\mathrm{CD} 11 \mathrm{~b}$ on the neutrophils was observed (Figure 3e). These experiments showed that the exclusive mode of action demonstrated by LPS-stimulated Treg cells towards neutrophils could not be explained by the greater surface expression of ICAM-1 on Treg cells or CD11b on neutrophils and that CD11b-ICAM-1-dependent interaction is important but not the only step triggering the induction of IL10-producing neutrophils.

\section{IL-10-producing neutrophils are apoptotic}

We have previously shown that CD3/CD28- and LPSstimulated Treg cells induce neutrophil apoptosis. ${ }^{13}$ To examine whether apoptotic neutrophils are the source of IL-10 following interaction with LPS-stimulated Treg cells, double staining with Annexin $\mathrm{V}^{+}$and anti-IL-10 mAbs was performed and the intensity of fluorescence was analyzed under a confocal microscope. The results indicated that the apoptosis of neutrophils induced by CD95L, unstimulated Treg cells, or CD3/CD28 mAb-stimulated Treg cells was not accompanied by increased IL-10 production (Figure 4a). On the contrary, LPS-stimulated Treg cells significantly augmented both apoptosis and IL-10 production by neutrophils, as about $70 \%$ of these cells were found double positive (Figure $4 \mathbf{b}$ ). The role of adhesion molecules was also confirmed in these analyses. Blocking CD11b on neutrophils and ICAM-1 on LPS- stimulated Treg cells caused partial inhibition of apoptosis of neutrophils (Figure 4a,b). Both neutralizing isotype controls were negative, as the fluorescence signal was on the same level as in neutrophil plus Treg (LPS) sample (Figure 4a). Collectively, interaction of CD11b with ICAM-1 is partially responsible for both induction of IL-10 production by neutrophils and apoptosis.

To show that apoptosis pathways are differently triggered by CD3/CD28 mAb-activated Treg cells and LPS-stimulated Treg cells, a profiling of the apoptosis-associated genes in the neutrophils was performed. A comparison of gene expression revealed that apoptosis induced by LPS-stimulated Treg cells was associated with a more than 10-fold upregulation of Apaf-1, Caspase 9, Bim, Puma, and Noxa, indicating activation of the mitochondrial/intracellular pathway of apoptosis (Figure 4c, see Supplementary Table S1 online). Overexpression of cytochrom $c$ protein in neutrophils cocultured with LPS-stimulated Treg cells compared with neutrophils cocultured with CD3/CD28-activated Treg cells additionally confirmed the engagement of mitochondrialmediated apoptosis (Figure $4 \mathbf{d}$ ).

\section{IL-10 feedback and apoptosis in neutrophils}

Our previous study showed that apoptosis of neutrophils induced by LPS-stimulated Treg cells was partially inhibited in the presence of anti-IL-10 neutralizing mAbs, although Treg cells were not the source of IL-10 under those particular experimental conditions. ${ }^{13}$ IL-10 has been already demonstrated to promote apoptosis in monocytes/macrophages, but it remains unknown whether IL-10 induces the same process in neutrophils exposed to LPS-stimulated Treg cells and in what way this takes place. ${ }^{18-20}$ To address this issue, anti-IL-10 blocking mAbs were added to the coculture of neutrophils and LPS-stimulated Treg cells, and significant inhibition of both apoptosis and IL-10 intracellular synthesis was noted (Figure 5a). Only a small subset of neutrophils $\mathrm{ANX}^{+} / \mathrm{IL}-10^{+}$ $(8 \%)$ or $\mathrm{ANX}^{-} / \mathrm{IL}^{-10^{+}}(12 \%)$ was detected at $5 \mathrm{~h}$ of incubation in blocking experiments (see Supplementary Figure S1). We 
a

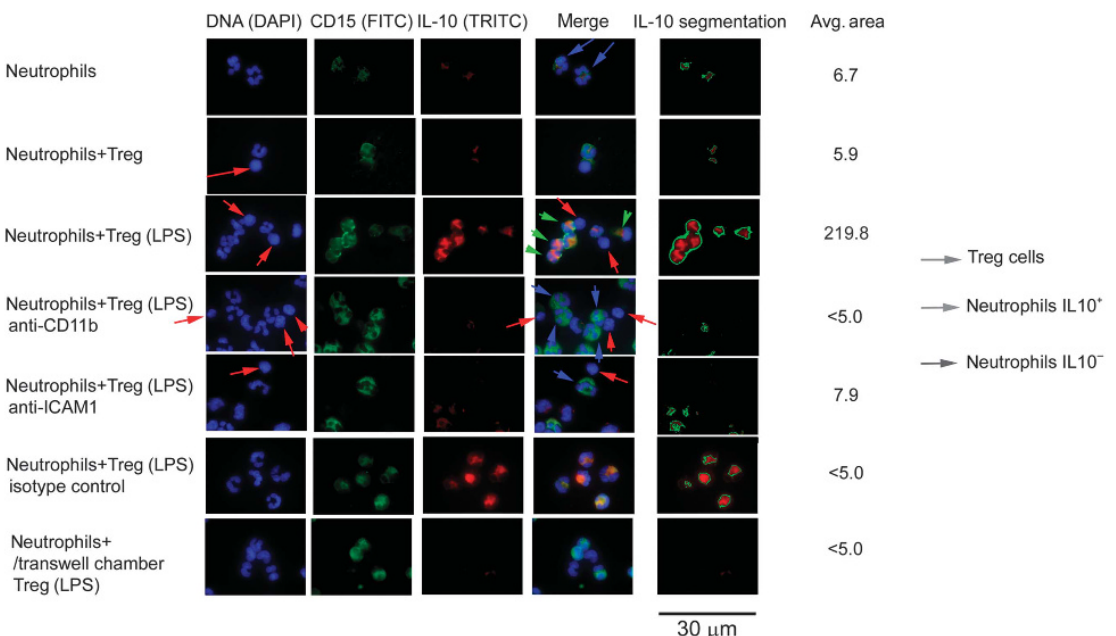

b
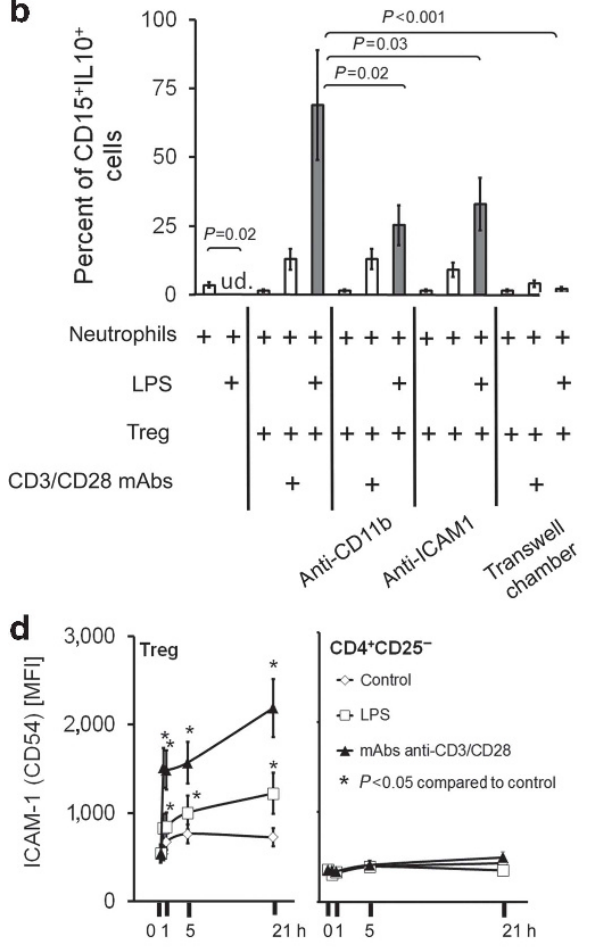

e

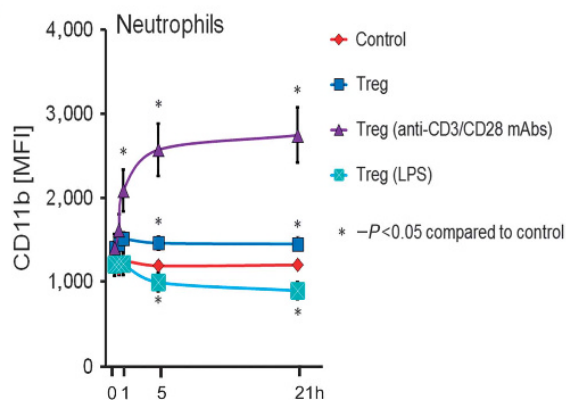

C
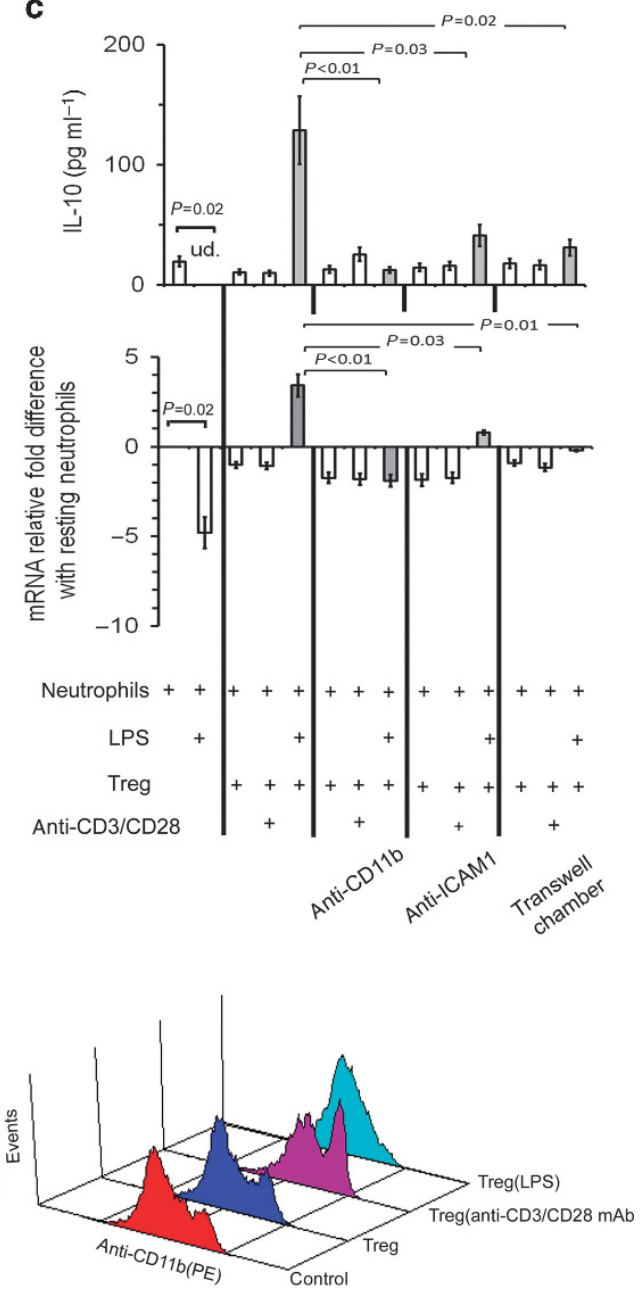

Figure 3 Polarization of neutrophils into interleukin (IL)-10-producing cells is dependent on the direct interaction with lipopolysaccharide (LPS)-stimulated regulatory T (Treg) cells via CD11b-ICAM-1. (a) Inhibition of IL-10-producing neutrophils in cocultures with anti-CD11b or intercellular adhesion molecule 1 (ICAM-1) blocking monoclonal antibodies (mAbs). (b) Quantitative analysis of CD15 ${ }^{+} \mathrm{IL}-10^{+}$neutrophils in the presence of anti-CD11b or ICAM-1 blocking mAbs. (c) Enzyme-linked immunosorbent assay analysis of extracellular levels of IL-10 and real-time reverse transcriptase-PCR analysis of IL-10 mRNA expression in neutrophils. (d) Induction of ICAM-1 expression on LPS- or anti-CD3/CD28 mAb-activated Treg cells as opposed to T conventional (Tconv) cells. Treg or Tconv cells were treated with $100 \mathrm{ng} \mathrm{ml}^{-1} \mathrm{LPS}$ or $5 \mu \mathrm{g} \mathrm{ml}^{-1}$ anti-CD3/CD28 mAbs for 0-48 $\mathrm{h}$, and ICAM-1 expression was analyzed at six time points by flow cytometry. (e) Downregulation of CD11b expression on neutrophils incubated with LPS-stimulated Treg cells. The example of flow cytometry analysis of neutrophils at $21 \mathrm{~h}$ of incubation (low right panel). Data are from four experiments. DAPI, 4,6-diamidino-2phenylindole; FITC, fluorescein isothiocyanate; MFI, mean fluorescence intensity; PE, phycoerythrin; TRITC, tetramethylrhod-amine isothiocyanate. 
a

Neutrophils

Neutrophils (CD95L)

Neutrophils + Treg

Nentrophils + Treg

Neutrophils + Treg

(anti-CD3/CD28 mAbs)

Neutrophils + Treg (LPS)

Neutrophils (anti-CD11b mAb) + Treg (LPS)

Neutrophils

+ Treg (LPS+anti-ICAM1 mAb)

Neutrophils (isotype control mAb) + Treg (LPS)

Neutrophils
+ Treg (LPS+isotype control mAb)
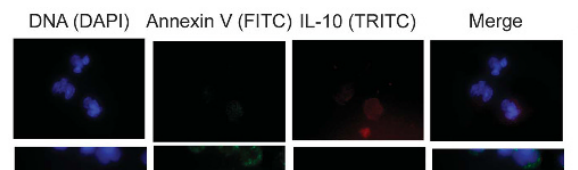

$\begin{gathered}\text { Annexin segmentation } \\ \text { avg. area }\end{gathered}$
$<5.0$

IL-10 segmentation

avg. area

10.6

21.8

$<5.0$

18.9

$<5.0$

12.4

$<5.0$

39.8

109.1
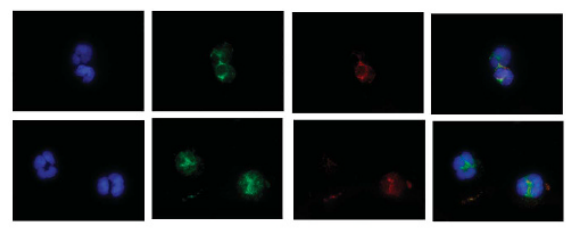

18.3

15.5

22.5
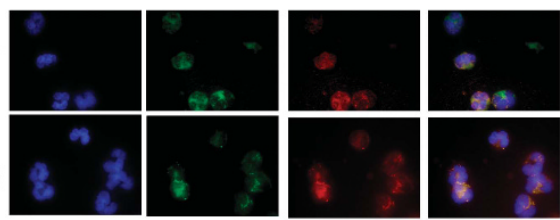

41.4

114.4

37.9

120.7

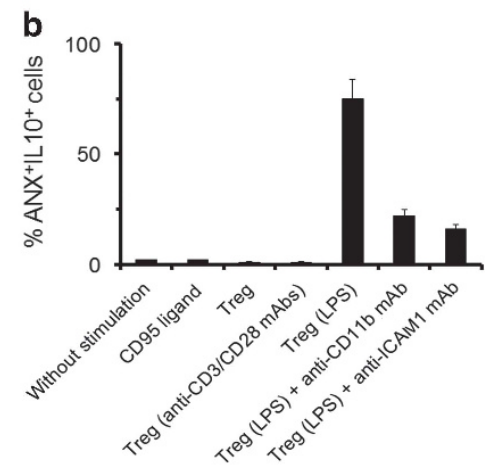

c

$30 \mu \mathrm{m}$

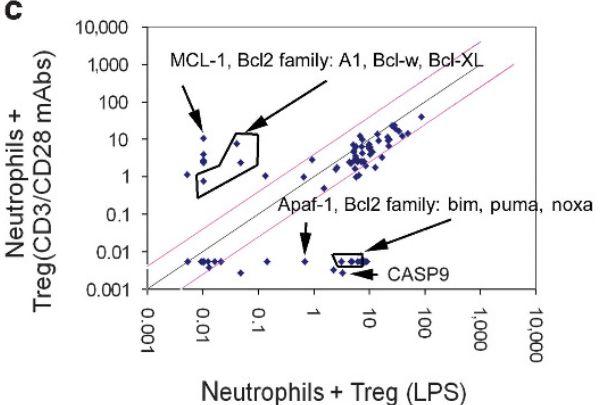

d

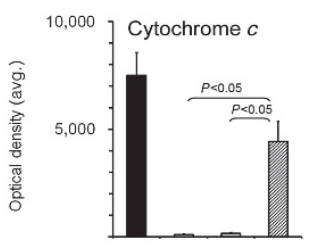

Cytochrome $c(15 \mathrm{kDa})$

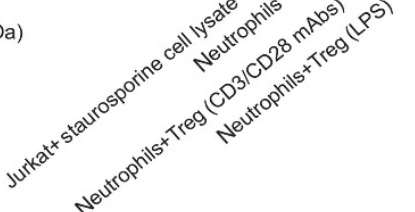

Figure 4 Interleukin (IL)-10-producing neutrophils induced by lipopolysaccharide (LPS)-stimulated regulatory T (Treg) cells are apoptotic (a) Neutrophils were stained with IL-10-TRITC (tetramethylrhod-amine isothiocyanate) and Annexin V-FITC (fluorescein isothiocyanate)and analyzed by immunocytochemistry. Data are representative of four experiments. (b) Quantitative analysis of $\mathrm{ANXV}^{+} \mathrm{IL}_{-10}{ }^{+}$neutrophils. The isotype control and secondary antibody incubated without first ab were negative. Data are from four experiments. (c) Comparison of apoptosis-related gene expression in neutrophils cocultured with LPS-stimulated Treg cells vs. CD3/CD28 monoclonal antibody (mAb)-stimulated Treg cells. Neutrophils cocultured with LPS-stimulated Treg cells underwent apoptosis triggered by mitochondrial pathway. The pink lines indicate thresholds of at least fourfold upregulation or downregulation of the respective gene. Data are representative of four independent experiments. (d) Upregulation of cytochrom $c$ in the neutrophils lysates after interaction with LPS-activated Treg cells. An example of the western blotting analysis. Jurkat + Staurosporine cell lysates were used as the positive control. DAPI, 4,6-diamidino-2-phenylindole. 
a

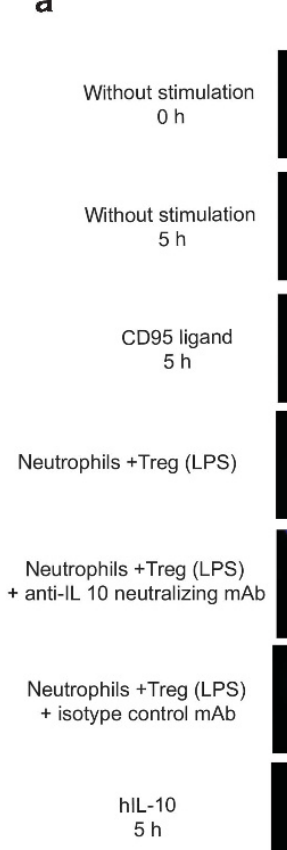

DNA (DAPI) Annexin V (FITC) IL-10 (TRITC)

Annexin segmentation IL-10 segmentation
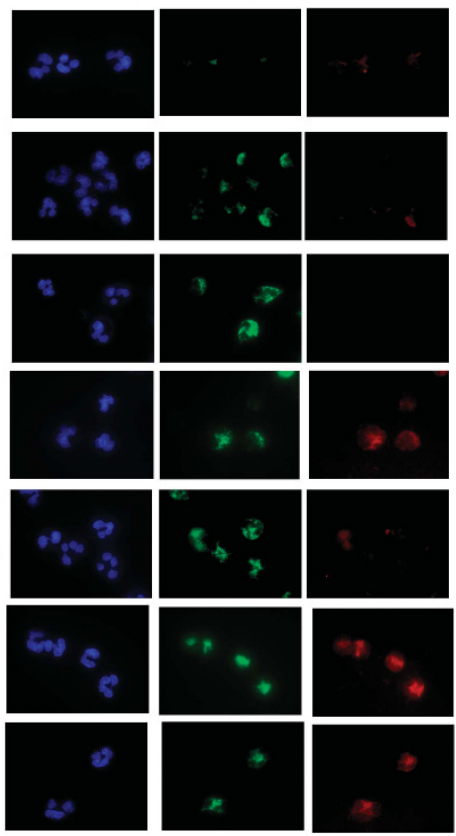

avg area

avg area
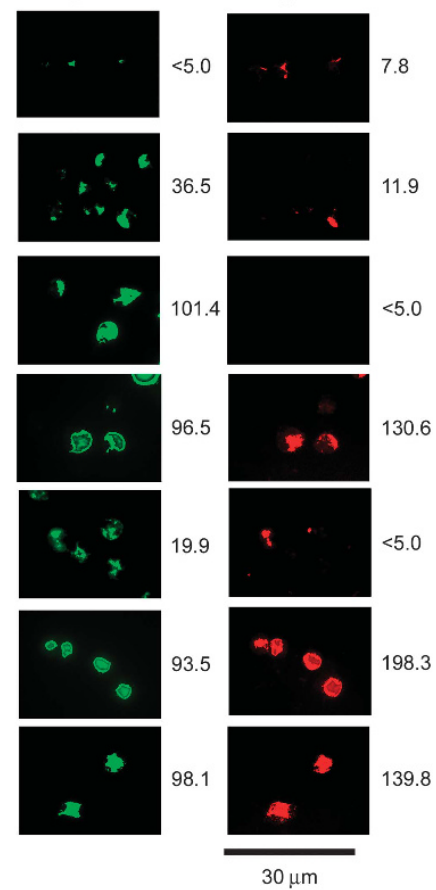

b

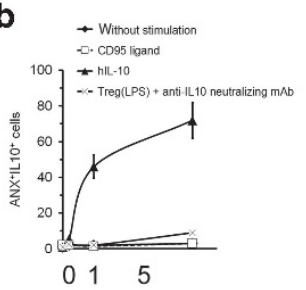

$21 \mathrm{~h}$
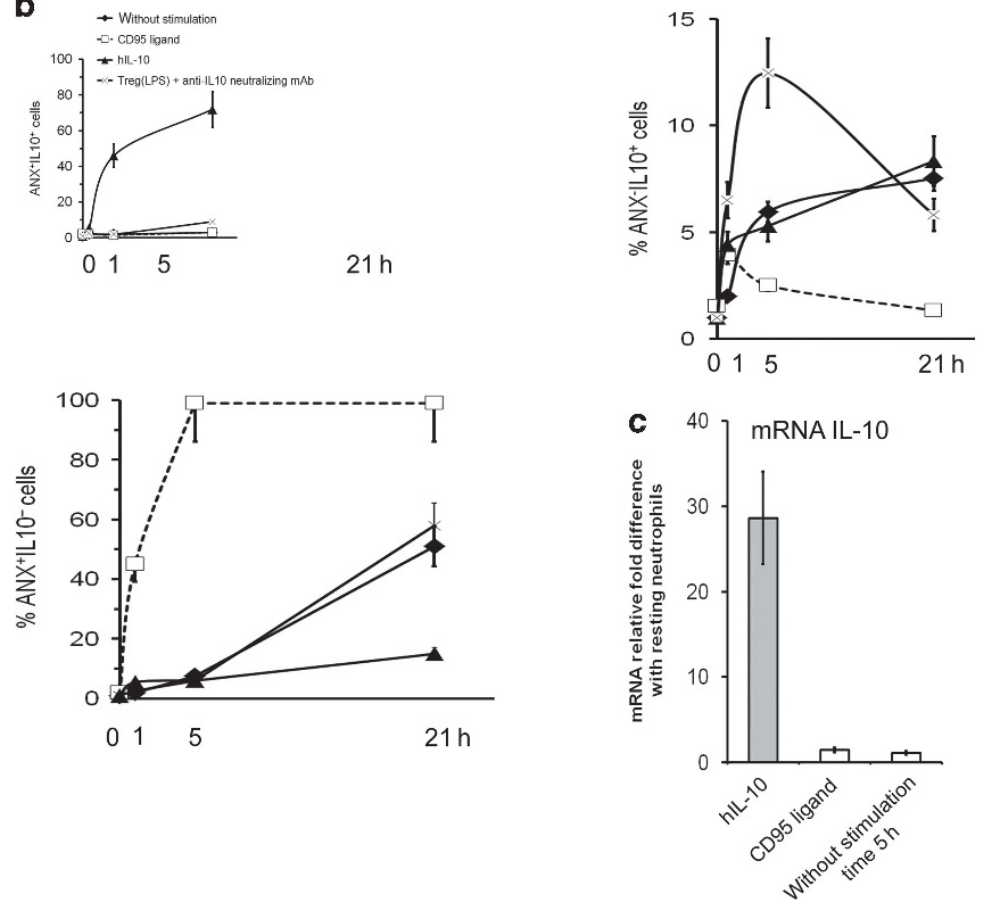

Figure 5 Interleukin (IL)-10 production and apoptosis of neutrophils is dependent on IL-10. (a) Neutrophils were stained with IL-10-TRITC (tetramethylrhod-amine isothiocyanate) and Annexin V-FITC (fluorescein isothiocyanate)in the presence of anti-IL10 blocking monoclonal antibodies (mAbs) or exogenous IL-10 and analyzed by immunocytochemistry. Data are representative of four experiments. (b) Quantitative analysis of $\mathrm{ANXV}^{+} \mathrm{IL}-10^{+}, \mathrm{ANXV}^{-} \mathrm{IL}-10^{+}$, and $\mathrm{ANXV}{ }^{+} \mathrm{IL}-10^{-}$neutrophils. (c) IL-10 mRNA quantitative analysis in 5-h incubations of neutrophils exposed to exogenous IL-10 or CD95L. Data are from four experiments. DAPI, 4,6-diamidino-2-phenylindole; LPS, lipopolysaccharide; Treg, regulatory T.

might assume that this subset of IL-10-positive neutrophils has been induced by direct interaction with LPS-stimulated Treg cells. We also found that the rate of ANX ${ }^{-} / \mathrm{IL}^{-}-10^{+}$neutrophils dropped at $21 \mathrm{~h}$ of incubation by about $10 \%$, whereas the rate of
$\mathrm{ANX}^{+} / \mathrm{IL}-10^{+}$neutrophils increased accordingly. Conversely, the treatment of neutrophils with exogenous IL-10 strongly induced both ANXV positivity and intracellular IL-10. Approximately $55-70 \%$ of neutrophils treated with IL-10 
were positive for ANXV and intracellular IL-10 at $5 \mathrm{~h}$ (see Supplementary Figure S1). The induction of IL-10 synthesis by exogenous IL-10 in neutrophils was further confirmed by real-time RT-PCR (Figure 5b).

To additionally address the possible autocrine/paracrine mode of action of IL-10, transwell and IL-10 receptor blocking experiments were performed. In the first one, we used transwell membrane that divided neutrophils from neutrophils incubated with LPS-stimulated Treg cell. To enable IL-10 to diffuse from neutrophil-LPS-stimulated Treg cell compartment to neutrophil compartment, cells were incubated for $21 \mathrm{~h}$. We found that nearly $71 \pm 13 \%$ of neutrophils separated by membrane were IL-10 positive (Figure 6a). In the next set of experiments, we used anti-IL10R neutralizing $\mathrm{mAb}$ prior to incubation of neutrophil with Treg cells. This experiment confirmed paracrine action of IL-10, as blocking of IL-10 receptor interrupted induction of IL-10 in neutrophils (Figure 6a). However, despite IL-10R blocking on neutrophils, a subset of IL-10-positive neutrophils was present
$(12 \pm 4 \%)$. These population was probably induced in the first phase by direct interaction with LPS-stimulated Treg cells. Neutralizing isotype control mAb was shown to be negative. Furthermore, we detected an expression of IL-10 receptor on the surface of freshly isolated neutrophils. Stimulation of neutrophils by LPS resulted in downregulation of IL-10RA (a decraese by $33 \%$ of non-stimulated neutrophils), in contrast to exogenous IL-10, which induced overexpression IL-10RA (a 25\% increase) (Figure 6b). These experiments highlighted a unique role of paracrine action of IL-10 in controlling neutrophil function and survival.

\section{LPS-stimulated Treg cells and hIL-10 contribute to the posttranslational chromatin modification of $\mathrm{H} 3$ histone associated with IL-10 locus gene in non-stimulated neutrophils}

Because induction of anti-inflammatory or proinflammatory gene expression is associated with chromatin remodeling and changes in histone modifications, we examined $\mathrm{H} 3$ histone

a
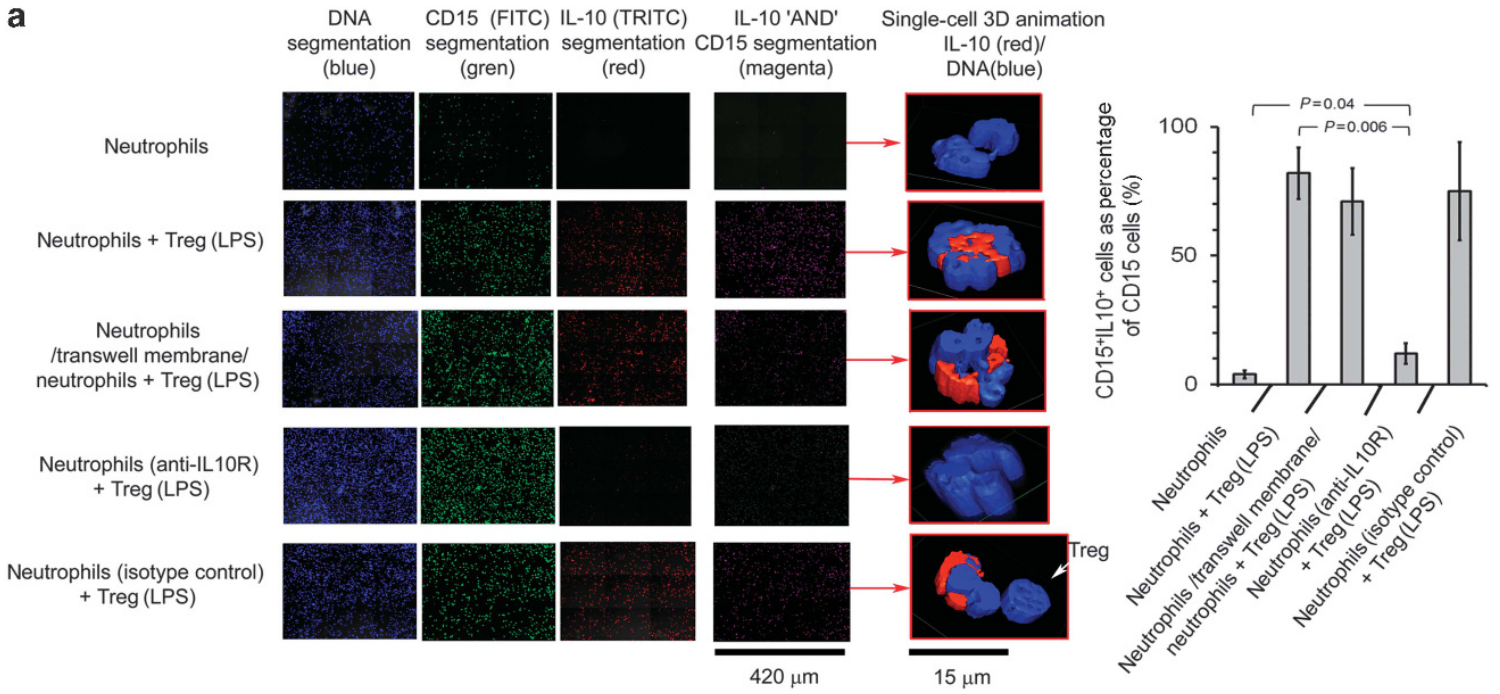

b
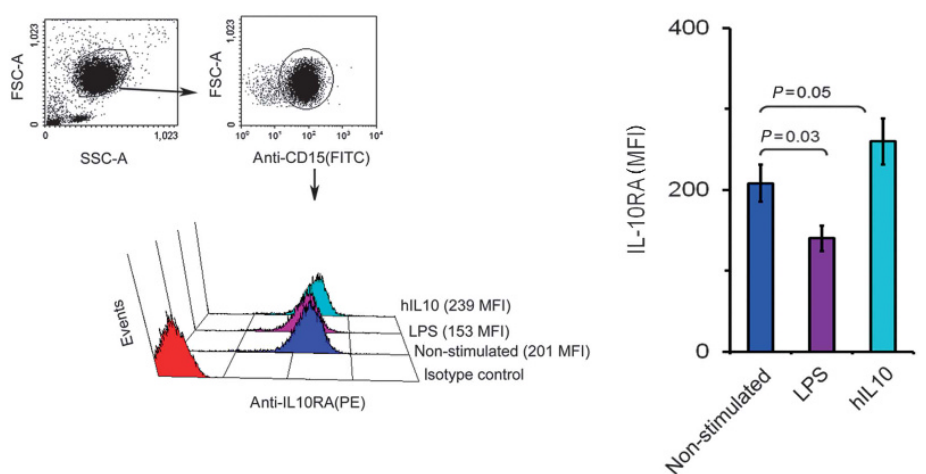

Figure 6 Two-step induction of interleukin (IL)-10 production by neutrophils. IL-10-producing neutrophils were first induced by interaction with lipopolysaccharide (LPS)-stimulated regulatory T (Treg) cells, and next IL-10 released by neutrophils boosted IL-10 production in a paracrine manner. (a) Quantitative analysis of IL-10 ${ }^{+}$neutrophils and 3D visualization of intracellular IL-10. Neutrophils were stained with IL-10-TRITC (tetramethylrhodamine isothiocyanate) in the presence of anti-IL10 receptors blocking monoclonal antibodies or after transwell incubation with neutrophils plus LPS-stimulated Treg cells and analyzed by immunocytochemistry. Data are representative of four independent experiments. (b) Expression of IL-10 receptor on neutrophils treated with LPS or hIL-10. Purified neutrophils were incubated with or without $100 \mathrm{ng} \mathrm{ml}^{-1} \mathrm{LPS}_{\mathrm{or}} 5 \mathrm{ng} \mathrm{ml} \mathrm{ml}^{-1} \mathrm{hIL-10}$ for $5 \mathrm{~h}$. Additional staining of cells with CD15 allowed to rule out possible monocyte contamination of neutrophils. Data are presented as mean fluorescence intensity $(\mathrm{MFI}) \pm$ s.d. $(n=4)$. FITC, fluorescein isothiocyanate; FSC, forward scatter; PE, phycoerythrin; SSC, side scatter. 
a

a Interleukin-10 precursor
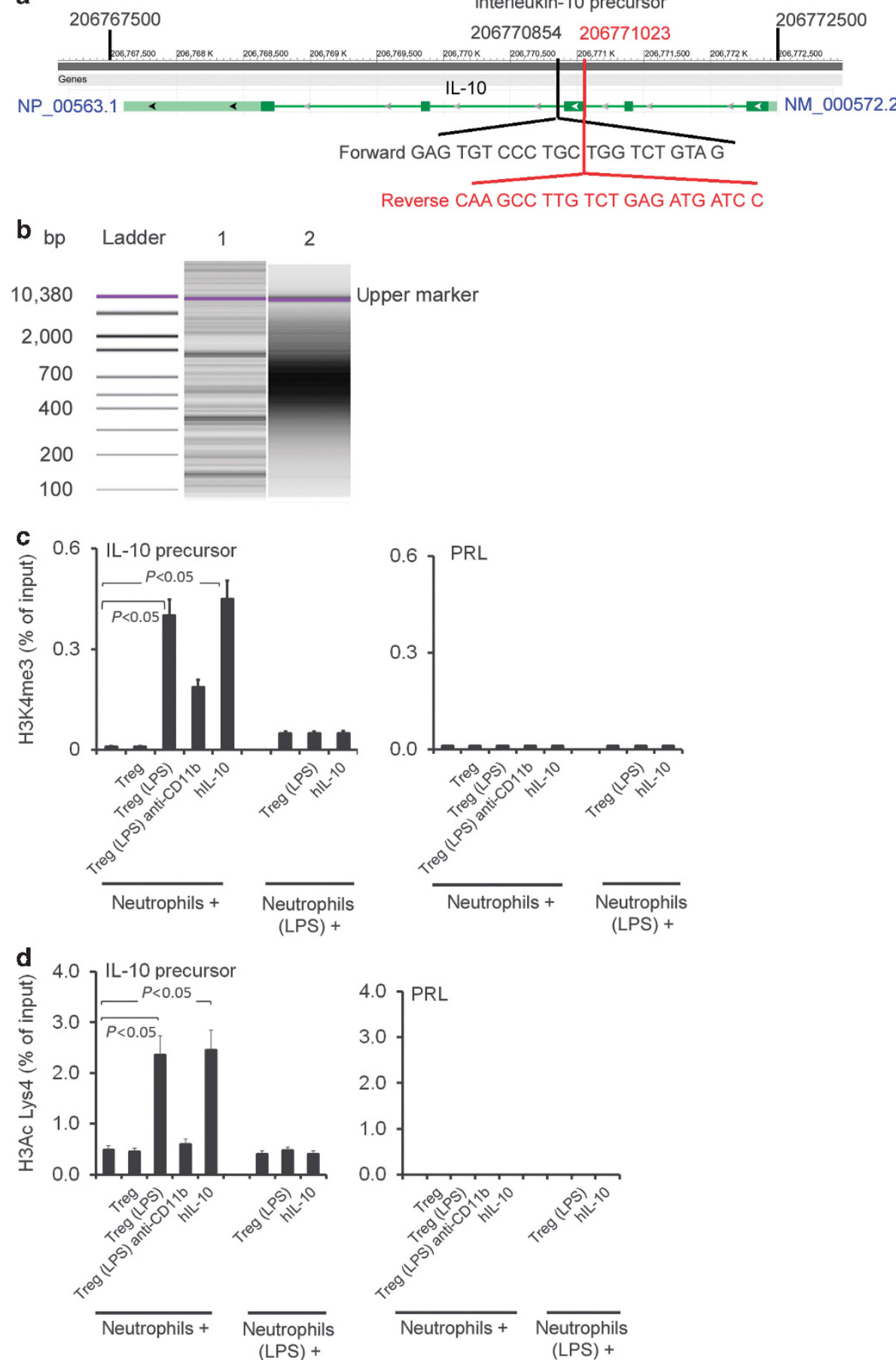

Figure 7 Lipopolysaccharide (LPS)-stimulated regulatory T (Treg) cells and exogenous human interleukin (IL)-10 exclusively promote chromatin modification at the $I L-10$ locus in human neutrophils. The ability to reorganize the chromatin at $I L-10$ locus is restricted to non-stimulated neutrophils. Neutrophils were incubated with Treg cells for $21 \mathrm{~h}$ and then negatively separated by magnetic beads and processed for chromatin immunoprecipitation (ChIP) assay. DNA samples precipitated by monoclonal antibodies were amplified using primer pairs specific for the precursor regions at the human $I L-10$ locus. (a) The position of the IL-10 precursor within the human IL-10 genomic locus. (b) The size of sonicated DNA checked by gel electrophoresis: 1-unsheared; 2-sheared. (c,d) H3K4me3 and H3Ac Lys4 ChIP analysis. Results are expressed as the percentage over an input DNA levels. PRL, prolactin.

status in $I L-10$ locus of neutrophils. We detected high levels of histone $\mathrm{H} 3$ trimethylated at $\mathrm{K} 4(\mathrm{H} 3 \mathrm{~K} 4 \mathrm{me} 3)$ and acetylated histone H3 (H3Ac Lys4) associated with transcriptionally active $I L-10$ gene in neutrophils incubated with LPS-stimulated
Treg cells or hIL-10 (Figure 7). Unstimulated or LPSstimulated neutrophils were negative for $\mathrm{H} 3 \mathrm{~K} 4 \mathrm{me} 3$ and H3Ac Lys4, which was in line with the previous findings. ${ }^{11}$ Preactivation of neutrophils with LPS blocked the induction of 
Periodontal abscess (gram-negative bacteria)
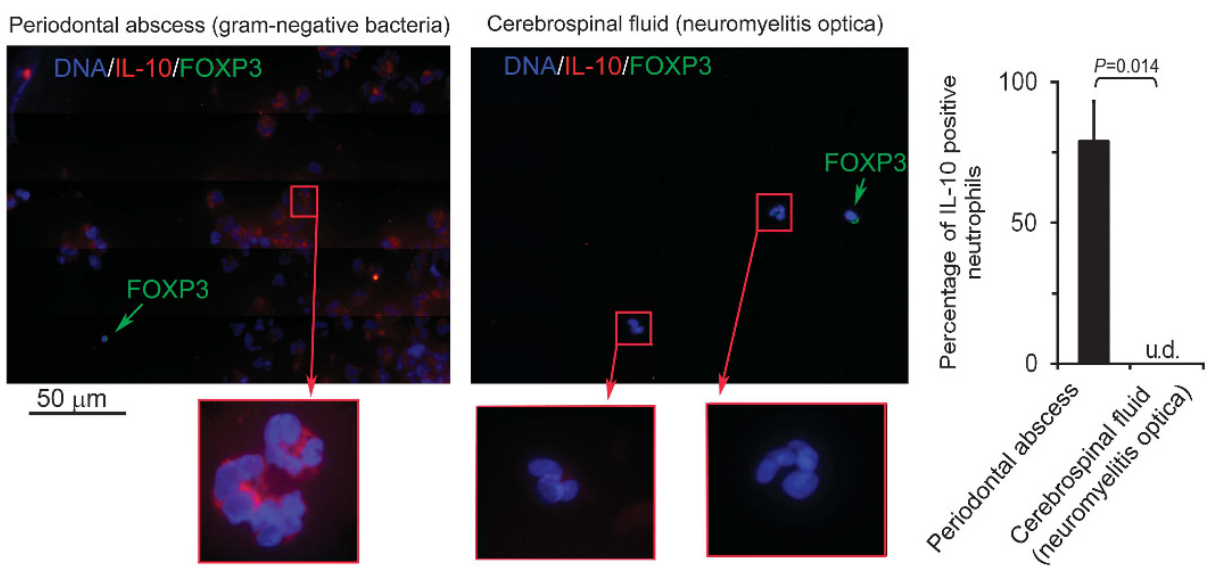

b

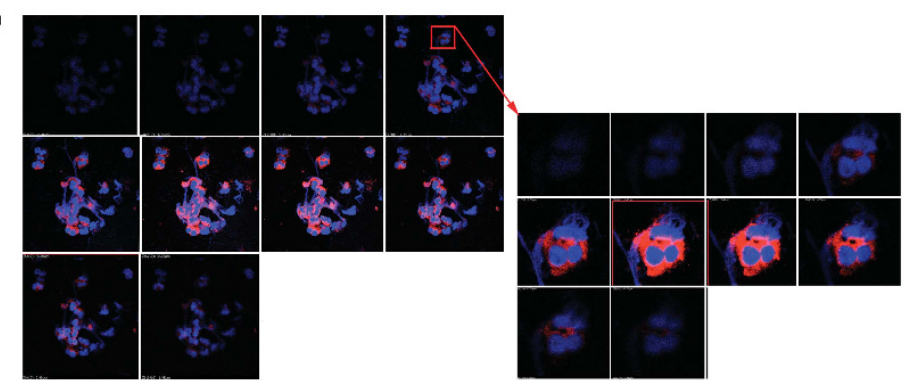

Figure 8 Detection of interleukin (IL)-10-producing neutrophils in vivo. (a) IL-10-positive neutrophils were identified in the purulent exudate from periodontal abscess caused by Gram-negative bacteria, contrary to IL-10-negative neutrophils present in the cerebrospinal fluid in aseptic inflammation. (b) Conformation of intracellular localization of IL-10 in z-stock analysis. ud., undetectable.

chromatin modifications in $I L-10$ locus during subsequent incubation with LPS-activated Treg cells or hIL-10 (Figure 7). Accordingly, our data indicate that chromatin at the $I L-10$ locus in unstimulated neutrophils is actively modified after interaction with LPS-stimulated Treg cells or exogenous human IL-10, but preactivation of neutrophils with LPS abolishes this process.

\section{Demonstration of IL-10-producing neutrophils in vivo}

To confirm that under certain conditions neutrophils are able to transform into IL-10-producing cells, we have chosen to seek for IL-10-positive neutrophils isolated from purulent exudate collected from periodontal pockets in patients with chronic periodontitis, a well-documented inflammation induced by Gram-negative bacteria. ${ }^{21}$ As a control model, we have chosen neutrophils isolated from the cerebrospinal fluid of patients with neuromyelitis optica (NMO) disease, an example of aseptic inflammation with neutrophil infiltration. ${ }^{22,23} \mathrm{We}$ found that FOXP3 ${ }^{+}$lymphocytes were present in the both types of collected material, whereas IL-10-positive neutrophils were detected only in the periodontal abscess exudate but not in the aseptic cerebrospinal fluid. The statistical analysis revealed that $79 \pm 14.2 \%$ of neutrophils present in the purulent exudate were IL-10 positive, in contrast to aseptic cerebrospinal fluid where IL-10-positive neutrophils were undetectable (Figure 8a). Confocal $z$-stock analysis displayed the intracellular localization of IL-10 in neutrophils collected from periodontal pockets (Figure $\mathbf{8 b}$ ). These experiments clearly confirm that human neutrophils are able to produce IL-10 during LPS-induced inflammation.

\section{DISCUSSION}

Neutrophils were considered to be a homogenous population for years. However, a few recent studies have addressed their potential heterogeneity in the context of their immunosuppressive capacity. ${ }^{8,9,11,14}$ Our work reports the identification of a subset of human IL-10-producing neutrophils to be present at the site of LPS-induced inflammation. Immunosuppressive neutrophils have been recently reported to appear in humans in vivo during acute systemic inflammation induced by endotoxin challenge or by severe injury. ${ }^{12,16}$ Murine neutrophils were found to produce IL-10. ${ }^{2-6}$ Moreover, in a variety of murine models of infectious diseases, cancer, bone marrow transplantation, and autoimmune diseases, neutrophils were shown to be a part of myeloid-derived suppressor cells. ${ }^{24-26}$

A great deal of controversy exists concerning the ability of human neutrophils to produce IL-10..$^{7-12,14,27-29}$ This discordance may result from different factors, such as purification procedures, culture protocols, and presence of contaminating monocytes/eosinophils. In our laboratory, we obtained reproducible data on IL-10 secretion by neutrophils, both at the mRNA and protein levels, using real-time RT-PCR, ELISA, and western blotting analysis. ${ }^{14}$ In the present study, we additionally used immunocytochemistry and chromatin 
immunoprecipitation (ChIP) analysis and showed that the presence of LPS-stimulated Treg cells or exogenous IL-10 significantly boosts IL-10 production by neutrophils, while anti-CD3/CD28 mAb-activated Treg cells or LPS have no such effect.

The mechanisms triggering IL-10 production in neutrophils are poorly investigated. Murine studies postulate that exposure to various microorganisms such as Gram-positive and Gramnegative bacteria, mycobacteria, Candida albicans, or Trypanasoma cruzi induces IL-10 secretion by neutrophils. However, in vitro findings demonstrate that only Toll-like receptors 2 and 4 agonists (Pam3CSK4 and LPS, respectively) trigger IL-10 production in neutrophils but at very low levels. ${ }^{2}$ Our results show that LPS at a low concentration $\left(100 \mathrm{ng} \mathrm{ml}^{-1}\right)$ had no effect on IL-10 production by neutrophils, but this concentration was sufficient for induction of specific Treg cells, which promote generation of IL-10-producing neutrophils. Serum amyloid A has been reported to systemically induce a population of IL-10-secreting neutrophils through binding to the G protein-coupled receptor FPR2 and activation of mitogen-activated protein kinase and phosphoinositide-3 kinase. $^{8}$ Another study demonstrated the appearance of a subset of suppressive neutrophils after intravenous administration of LPS in humans. ${ }^{16}$ Our study suggests that local induction of IL-10-producing apoptotic neutrophils may take place at the site of LPS-induced inflammation.

Visualization of ICC and differential interference contrast images of cocultured neutrophils and Treg cells allowed identification of the proximity of these cells, indicating direct cell-cell interaction. The ability of activated Treg cells to produce IL-8, as identified in a recent report, might be responsible for attracting neutrophils and facilitating direct cell-cell contact. ${ }^{17}$ Another recent study also revealed the ability of neutrophils to directly interact with $\mathrm{T}$ cells via CD11b. ${ }^{16}$ Here we demonstrated that pretreatment of Treg cells with LPS or anti-CD3/CD28 mAbs results in the upregulation of ICAM-1; however, TCR activation has greater effect. Subsequent incubation of pretreated Treg cells with neutrophils resulted in differential surface expression of CD11b. CD11b downregulation on neutrophils incubated with LPS-stimulated Treg cells might be a result of induction of apoptosis of neutrophils. ${ }^{30}$ Blocking experiments confirm that both $\mathrm{CD} 11 \mathrm{~b}$ on neutrophils and ICAM-1 on LPS-stimulated Treg cells are involved in neutrophil-Treg cell interactions that contribute to the induction of IL-10 production and apoptosis, but CD11b or ICAM-1 blockade has only partial effect on the IL-10 induction. However, transwell experiments definitely showed that direct cell-cell contact is required to induce IL-10 in neutrophils by LPS-stimulated Treg cells.

Although these results confirm that the direct interaction of neutrophils with LPS-stimulated Treg cells is crucial for induction of IL-10, they do not explain how such a small number of Treg cells incubated with neutrophils (1 to 50 ratio) was able to induce IL- 10 production in nearly $80 \%$ of cocultured neutrophils. It was plausible that IL-10 released by the neutrophils, which directly interacted with LPS-stimulated
Treg cells, might subsequently induce IL-10 production in neutrophils in a paracrine manner. The two-step IL-10 induction was confirmed by a set of different experiments: IL-10 neutralization, incubation of neutrophils with exogenous hIL-10, induction of IL-10 in neutrophils separated from autologous neutrophils incubated with LPS-stimulated Treg cells by transwell membrane, and neutralization of IL-10 receptors. IL-10 and IL10RA neutralizing experiments showed that direct interaction of neutrophils with LPS-stimulated Treg cells is responsible for induction of a relatively small subset of IL-10-positive neutrophils ( $\sim 20 \%)$. Paracrine IL-10 seems to be responsible for further $\sim 60 \%$ increase of IL-10-producing neutrophils. We additionally confirmed IL-10R expression on the human neutrophils, which is differentially regulated by LPS and IL-10.

Our in vitro findings reveal that neutrophils might acquire their anti-inflammatory properties at the site of LPS-induced inflammation, following the interaction with LPS-stimulated Treg cell by the CD11b-ICAM-1 binding, and subsequent acquisition of IL-10-producing ability in the second phase, which is dependent on the paracrine secretion of IL-10. To address the relevance of these findings in vivo, IL-10-producing neutrophils were identified at the site of inflammation induced by Gram-negative bacteria. Numerous IL-10-positive neutrophils and a few FOXP3 ${ }^{+}$T lymphocytes were found in the purulent exudates from the patients with chronic periodontitis, contrary to the presence of IL-10-negative neutrophils identified in the cerebrospinal fluid of patients with Devic disease. These observations confirm the ability of human neutrophils to transform into suppressor cells, which produce IL-10 during inflammation induced by Gram-negative bacteria.

IL-10 production induced by LPS-stimulated Treg cells or IL-10 itself was accompanied by increased numbers of apoptotic neutrophils. CD11b-ICAM-1 interaction might promote both apoptosis and neutrophil survival depending on the presence of additional Mac-1 ligands or proapoptopic factors, such as FasL and tumor necrosis factor. ${ }^{31}$ Double staining of neutrophils with ANXV and IL-10 confirmed that the majority of IL-10-producing cells are apoptotic. IL-10 has been shown to promote apoptosis of human monocytes and to have a counter-regulatory effect on the LPS-induced apoptosis of human neutrophils. ${ }^{19,20,32-34}$ The apoptosis of neutrophils is crucial for the resolution phase of inflammation: apoptotic neutrophils are then phagocytosed by macrophages or dendritic cells, which triggers anti-inflammatory properties in these phagocytes. ${ }^{34}$ The results of our study indicate that LPS-stimulated Treg cells, but not CD95L or TCR-activated Treg cells, are responsible for the induction of apoptotic neutrophils, which produce IL-10. Therefore, our study indicates that CD11b binding by ICAM-1 and subsequent IL-10 production facilitates neutrophil apoptosis. Lack of IL-10 synthesis in the experimental settings with the anti-CD3/CD28 $\mathrm{mAb}$-stimulated Treg might be a results of perforin/granzymedependent induction of apoptosis. ${ }^{13}$

An analysis of the apoptosis-associated genes expressed by the neutrophils was performed to clarify the molecular 
mechanism behind this phenomenon. The gene profile revealed that different pathways are activated depending on whether the neutrophils were incubated with LPS-stimulated Treg cells or Treg cells stimulated via TCR. The strong overexpression of Caspase 9, Apaf-1, Bim, Puma, and Noxa genes and cytochrome $c$ protein identified in neutrophils cocultured with LPS-stimulated Treg cells suggests engagement of the intracellular/mitochondrial pathway of apoptosis. Mitochondrial apoptosis occurs when the concerted action of the proapoptotic $\mathrm{Bcl}-2$ proteins lead to the permeabilization of the outer mitochondrial membrane, permitting the release of cytochrome $c$ from the intermembrane space into cytosol, where it binds Apaf-1, initialing caspase activation. ${ }^{35-37}$ Caspase- 9 is the essential initiator of the caspase cascade required for apoptosis signaling through the mitochondrial pathway. ${ }^{35}$ In the present study, we have shown that up to $70 \%$ of apoptotic neutrophils were positive for intracellular IL-10, which suggests a possible relationship between signals triggering mitochondrial apoptosis and IL-10 synthesis in human neutrophils. At this point, it is difficult to clearly state whether apoptosis and induction of IL-10 synthesis in neutrophils are linked. In IL-10 neutralizing experiments, a small subset of IL10-producing neutrophils was not apoptotic, and CD11b/ ICAM-1 interaction blockade seems to have greater effect on IL-10 synthesis than apoptosis. Therefore, induction of apoptosis in neutrophils incubated with LPS-stimulated Treg cells might be related both to direct CD11b/ICAM-1-dependent interaction, autocrine/paracrine effect of IL-10, and other undefined mechanisms.

It was recently demonstrated that chromatin at the IL-10 locus was inactive in human neutrophils stimulated with proinflammatory factors LPS, Pam3, CSK4, and SAA, contrary to human monocytes and murine neutrophils. ${ }^{11}$ However, it seems plausible that anti-inflammatory Treg cells and IL-10 might have an opposite effect. Indeed, we demonstrated that under certain conditions chromatin modification specific for transcriptionally active $I L-10$ gene is induced in human neutrophils. We detected higher levels of H3K4me3 and H3AcLys4 that reflect transcriptional activation of $I L-10$ precursor genes in unstimulated neutrophils incubated with LPS-stimulated Treg cells or hIL-10. However, neutrophils previously activated with LPS were insensitive to induction of chromatin modification in $I L-10$ locus by LPS-stimulated Treg cells or hIL-10. LPS stimulation irreversibly switches neutrophils into proinflammatory cells and blocks the chromatin modification at the $I L-10$ locus, which is in accordance with the pervious findings. ${ }^{11}$ However, the results of these experiments do not fully correspond with ICC experiments, in which unstimulated neutrophils produced detectable amounts IL-10, and nearly 7\% cells were IL-10 positive (Figure 1a,b). This discrepancy probably is a result of different sensitivity of methods and/or variations of human material used in experiments. ICC analysis is more sensitive compared with western blotting or mRNA analysis. In ICC, we are able to analyze at the level of single cell, contrary to western blotting or quantitative mRNA where at least 1-5 millions of positive cells are needed. Additionally, the chromatin analysis showed detectable amounts of H3AcLys4 that reflect transcriptional activation of $I L-10$ precursor genes in unstimulated neutrophils, while prolactin, a negative control, was undetectable.

Our findings are in line with the concept of regulatory functions of neutrophils, which are not a homogenous population of effector cells. Furthermore, our experiments support the idea of different models of action of Treg cells, depending on whether they operate in non-inflammatory or inflammatory conditions. ${ }^{38}$ At sites that are constantly exposed to invading microbes (intestinal mucosa), Treg cells employ IL10 to control a self-damaging excessive immune response not only by producing it themselves but also by inducing production in the populations of other IL-10-producing cells, such as Tr1 cells, monocytes, and neutrophils. This might also explain the contradictory results observed in studies assessing the possibility of neutrophils to synthesize IL-10. In the majority of experiments with negative results, neutrophils were treated with proinflammatory stimuli, such as fMLP, interferon- $\gamma$, tumor necrosis factor, granulocyte-macrophages colony-stimulating factor, granulocyte colony-stimulating factor, LPS, Pam3CSK4, poly(I:C), or R-848. ${ }^{10,27,28}$ In macrophages, LPS not only triggers nuclear factor- $\kappa \mathrm{B}$ activation and subsequent production of proinflammatory cytokines but also initiates the negative feedback loop associated with IL-10 synthesis via a Janus-activated kinase/signal transducer and activator of transcription factor 3-dependent pathway. ${ }^{39}$ The different ability of human monocytes and neutrophils to secrete IL-10 may be accounted for the differences in the chromatin status of the $I L-10$ locus. ${ }^{11}$ The different plasticity of neutrophils and macrophages might be a consequence of the different lifespans of these cells. Neutrophils, as short-lived cells, are not probably able to switch from a proinflammatory to an anti-inflammatory character, as monocytes/macrophages do. According to our previous and present studies, Treginduced apoptosis and IL-10 production occurs in freshly isolated neutrophils but not in those that have been preactivated by LPS which might suggest that neutrophils could be polarized into proinflammatory or anti-inflammatory cells. However, this effect is irreversible. These results might facilitate a better understanding of the pathogenesis of sepsis, which is characterized by the impairment of neutrophil migration into the infection site, also termed neutrophil paralysis. During sepsis, LPS affects circulating neutrophils. The preactivation and subsequent activation of neutrophils by LPS results in a prolonged lifespan of these cells by inhibition of apoptosis and prevents their transformation into an IL-10-producing cell (Figure 9).

In conclusion, the data presented here clearly demonstrate the ability of human neutrophils to produce IL-10 at the site of LPS-induced inflammation, in response to direct interaction with LPS-activated Treg cells in the first phase and paracrine effect of IL-10 in the second phase. We confirmed the IL-10 synthesis pathway starting from DNA activation (ChIP analysis) through transcription (IL-10 mRNA) and translation (ICC analysis and western blotting) up to the release of IL-10 

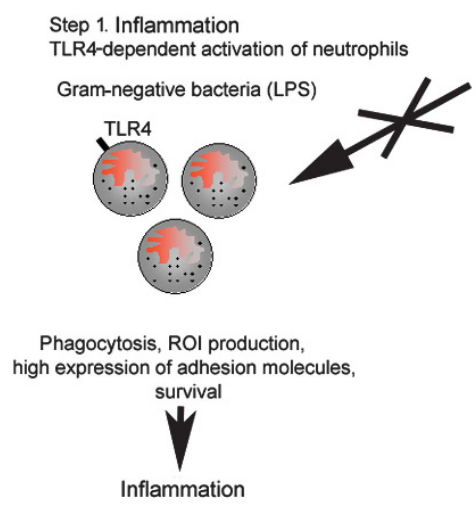

Step 2. Recruitment of treg cells Treg cell

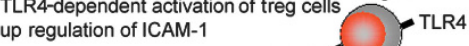

up regulation of ICAM-1

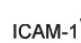

Step 3.Interacton of LPS-activated treg with non-activated neutrophils CD11b-ICAM1 synapse

induction of apoptosis and IL-10-producing neutrophils

Neutrophil - Treg cell

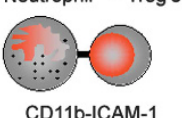

Step 4.IL-10-producing neutrophils induce apoptotic IL-10-producing neutrophils

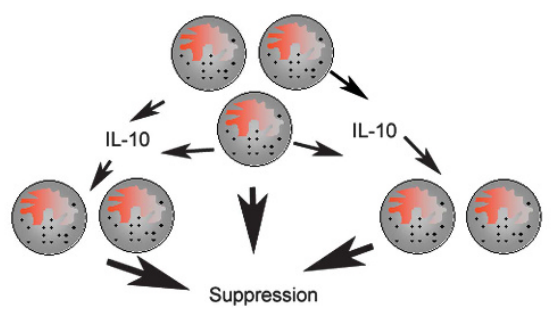

Figure 9 The role of neutrophils and regulatory T (Treg) cells in lipopolysaccharide (LPS)-induced inflammation and subsequent suppression. At the beginning, LPS induces effector functions and survival of neutrophils facilitating elimination of bacteria. Next, Treg cells recruited to the site of inflammation are activated by LPS and subsequently interact with newly migrated neutrophils providing an inhibitory signal. As a consequence, neutrophils produce interleukin (IL)-10 and undergo apoptosis. IL-10 released by neutrophils, in a positive feedback loop manner, induces IL-10 production and apoptosis in other neutrophils. The reaction might continue until all neutrophils are transformed into apoptotic bodies, facilitating subsequent phagocytosis by macrophages and resolution of inflammation. It is important to note that LPS-stimulated Treg cells have an inhibitory effect only when interacting with non-activated neutrophils. Previous activation of neutrophils with LPS desensitizes them to Treg cell influence. ICAM-1, intercellular adhesion molecule 1; ROI, reactive oxygen intermediates; TLR, Toll-like receptor.

into intracellular space (ELISA). Further investigations based on these observations may result in finding therapeutic means to reinforce immunity toward inflammation caused by multidrug-resistant Gram-negative bacteria.

\section{METHODS}

Neutrophils isolation. The neutrophils were isolated in LPS-free conditions on Polymorphprep (Axis Shield, Oslo, Norway) by centrifugation of freshly drawn blood from the same donors as $\mathrm{T}$ cells at $450 \mathrm{~g}$ at $20^{\circ} \mathrm{C}$ for $30 \mathrm{~min}$, followed by erythrocyte lysis with ammonium chloride buffer at $20^{\circ} \mathrm{C}$ for $5 \mathrm{~min}\left(155 \mathrm{mM} \mathrm{NH}_{4} \mathrm{Cl}, 10 \mathrm{~mm}\right.$ $\mathrm{KHCO}_{3}$, and $0.1 \mathrm{~mm}$ EDTA; BD Pharmingen, San Jose, CA).

For ChIP analysis, mRNA expression analysis, and mRNA profiling, cells were isolated using negative selection (MACSxpress Neutrophil Isolation Kit, Miltenyi Biotec, Bergisch Gladbach, Germany) according to manufacturer's instruction, with minor modification (we used sodium heparin $10 \mathrm{IU} \mathrm{ml}^{-1}$ instead of EDTA).

To determine the purity and viability of isolated neutrophils, staining with $\mathrm{mAbs}$ to $\mathrm{CD} 16, \mathrm{CD} 15, \mathrm{CD} 14$, and 7-AAD (7aminoactinomycin D) was performed using flow cytometry (LSRII, $\mathrm{BD}$, San Jose, CA) and BD FACSDiva analysis software. The following mAbs were used: CD14-PE (clone M5E2), CD15-FITC (fluorescein isothiocyanate) (MMA), and CD16-PECy7 (3G8, all from BD Pharmingen). Flow cytometric analysis of isolated population of cells showed that the percentage of $\mathrm{CD} 15^{\text {high }} \mathrm{CD} 16^{+} \mathrm{CD} 14^{-}$neutrophils was $\sim 97 \%$. The level of contaminating $\mathrm{CD} 14^{+} \mathrm{CD} 15^{+}$monocytes was about $1.4 \%$ and $\mathrm{CD} 15^{+} \mathrm{CD} 16^{-}$eosinophils was $<0.5 \%$ after isolation on the Polymorphprep. Nearly $100 \%$ of cells were viable (7-AAD negative) (see Supplementary Figure S2).

Neutrophils were incubated without stimulation, in the presence of $100 \mathrm{ng} \mathrm{ml}^{-1}$ ultrapure LPS from E. coli (serotype R515, Alexis Biochemicals, San Diego, CA), $1 \mu \mathrm{g} \mathrm{ml}^{-1}$ anti-CD95 ligand $\mathrm{mAb}$
(CH-11 IgM, Immunotech, Marswille, France, $5 \mathrm{ng} \mathrm{ml}^{-1}$ human IL10 (expressed in Sf21 insect cells, Sigma-Aldrich, St. Louis, MO), or with either unstimulated, LPS- or CD3/CD28-stimulated CD4 ${ }^{+}$ $\mathrm{CD} 25^{+} \mathrm{CD} 127^{\text {low }}$ Treg or Tconv cells, respectively, at a ratio of 50:1 (neutrophils:T cells) in RPMI 1640 culture medium (described above) for $0.5-21 \mathrm{~h}$. Alternatively, neutrophils and Treg cells were incubated in the transwell chambers using Nunc Cell Culture inserts (pore size $0.02 \mu \mathrm{m}$, Nunc, Roskilde, Denmark). In transwell experiment, plates were incubated on the orbital shaker (Biosan, Riga, Latvia 50 r.p.m.).

For quantitative immunofluorescence analysis, cells were cultured on biocoat 96-well black/clear plate coated by poly-D-Lysine cellware (Becton Dickinson, San Jose, CA). For fluorescent intensity analysis of IL-10, cocultured cells were transferred from U-bottom plates (Nunc) to gelatin-coated microscope slides by cytospine. The visualization of interactions of live neutrophils with Treg cells was performed on the glass chamber slides (Nunc).

To analyze the role of CD11b and ICAM-1 in the interactions of neutrophils and Treg cells, neutrophils were treated with anti-CD11b neutralizing mAbs ( $1 \mu \mathrm{g} \mathrm{ml}^{-1}$, ICRF44(44); BD Pharmingen) for 10 min prior to addition to Treg cells or Treg cells were treated with anti-ICAM-1 neutralizing pAbs $\left(10 \mu \mathrm{g} \mathrm{ml}^{-1}\right.$ sheep IgG, R\&D Systems, Minneapolis, MN) for $10 \mathrm{~min}$ before addition to neutrophils. To examine the effect of autocrine/paracrine IL-10 in induction of IL10-positive neutrophils, we treated neutrophils with anti-IL10RA neutralizing mAbs $\left(1 \mu \mathrm{g} \mathrm{ml}^{-1}, 37607.11\right.$, Abcam, Cambridge, UK) for $10 \mathrm{~min}$ prior to addition to Treg cells. As the control antibody, IgG1 (clone 107.3; BD Pharmingen) and purified sheep IgG (R\&D Systems) respectively, were used.

T-cell isolation. Peripheral blood mononuclear cells were isolated by density centrifugation over Lymphoprep (Axis-Shield, Oslo, Norway). Human $\mathrm{CD}^{+}{ }^{+} \mathrm{T}$ cells were preenriched from peripheral blood mononuclear cells with a CD4-negative Cell Isolation Kit (Miltenyi Biotec) and an AutoMACS cell sorter (Miltenyi Biotec, Bergisch 
Gladbach, Germany) using the double column magnetic separation procedure. Isolated CD4 T cells were stained with fluorescent-labeled mAbs directed against human CD4-PerCP (clone SK3), CD25-FITC (2A3) and CD127-PE (hIL-7R-M21, all provided by BDPharmingen) for $30 \mathrm{~min}$ at $4{ }^{\circ} \mathrm{C}$. $\mathrm{CD} 4{ }^{+} \mathrm{CD} 25^{\text {high }} \mathrm{CD} 127^{\text {low }}$ and $\mathrm{CD} 4{ }^{+} \mathrm{CD} 25^{-}$ subsets were isolated by FACS (fluorescence-activated cell sorter) sorting procedure using a FACSAria with purity mask mode (BD Biosciences, San Jose, CA). The purity of FACS-sorted CD4 ${ }^{+}$ $\mathrm{CD} 25^{+} \mathrm{CD} 127^{\text {low }}$ and $\mathrm{CD} 4{ }^{+} \mathrm{CD} 25^{-}$cell fraction assessed by threecolor flow cytometry was $\sim 99 \%$. The $\mathrm{T} \mathrm{CD} 4^{+} \mathrm{CD} 25^{\text {high }} \mathrm{CD} 127^{\text {low }}$ regulatory (Treg) or T CD $4{ }^{+} \mathrm{CD} 25^{-}$conventional (Tconv) cells were

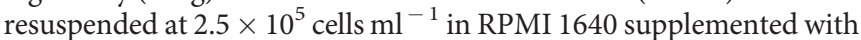
$10 \%$ heat-inactivated fetal calf serum (Gibco, Waltham, MA), 2 mM L-glutamine (Gibco), and 1\% penicillin/streptomycin (Sigma-Aldrich) in 96 sterile (nonpyrogenic) polysterene U-bottom plates (BD Biosciences) in the presence or absence of $100 \mathrm{ng} \mathrm{ml}^{-1}$ ultrapure LPS from E. coli (serotype R515, Alexis Biochemicals) or $5 \mu \mathrm{g} \mathrm{ml}^{-1}$ antiCD3 (HIT3a; BD Pharmingen) with $5 \mu \mathrm{g} \mathrm{ml}^{-1}$ anti-CD28 (CD28.2; BD Pharmingen) mAbs, respectively, for $48 \mathrm{~h}, 37^{\circ} \mathrm{C}, 5 \% \mathrm{CO}_{2}$. Before incubation with the neutrophils, the Treg cells were washed in phosphate-buffered saline containing $\mathrm{Ca}^{+2} / \mathrm{Mg}^{+2}$.

\section{Analysis of IL-10-positive neutrophils in vivo}

NMO disease. Six patients were diagnosed with NMO disease at the Department of Neurology, Medical University of Lodz, Lodz, Poland and fulfilled the 1999 Wingerchuk criteria for NMO ${ }^{40}$ All the patients had optic nerve and spinal cord inflammation; none of them had brain magnetic resonance imaging that fulfilled the Barkhof/Tintore criteria for multiple sclerosis, all were Aquaporine 4 autoantibody positive. ${ }^{41}$ The $350 \mu$ of fluid core was cytospined $(300 \mathrm{~g}, 30 \mathrm{~min})$ on the gelatincoated microscope slides and fixed by $4 \%$ paraformaldehyde for $20 \mathrm{~min}$ in room temperature.

Periodontal abscess. Five patients with untreated chronic periodontitis and single periodontal abscesses were diagnosed at the Department of Periodontology and Oral Mucosal Diseases, Medical University of Lodz. Purulent exudates were collected from the periodontal pocket before any mechanical or pharmacological treatment was performed. One drop of purulent exudate was applied on microscope slides and fixed by $4 \%$ paraformaldehyde.

To visualize IL-10-positive neutrophils and Treg cells in both groups of patients, double staining with anti-IL-10 and anti-FOXP3 mAbs (PE, clone 206D, BioLegend, San Diego, CA) was used. Neutrophils were identified based on the morphological features of the nucleus. Images were acquired using BD Pathway Bioimager 850 (San Jose, CA) and confocal Nikon D-Eclipse C1 (Tokyo, Japan) microscopes.

The experimental protocol was approved by the Ethics Committee of the Medical University of Lodz, and informed consent was obtained from all participants of the study.

Statistical analysis. Arithmetic means and s.ds. were calculated for all parameters in at least four independent experiments. A statistical analysis of differences was performed using the one-way analysis of variance test. Scheffe's test was used for multiple comparisons as a post-hoc test when statistical significances were identified in the analysis of variance test. Statistical analysis between NMO disease patients and periodontal abscess from chronic periodontitis patients was counted by Student's $t$-test. Statistical significance was set at $P<0.05$.

Flow cytometry, microscopy, western blotting, ELISA, real-time quantitative PCR, multiple gene profiling microarray, and ChIP assays. See Supplementary Experimental Procedures for details.

SUPPLEMENTARY MATERIAL is linked to the online version of the paper at http://www.nature.com/mi

\section{ACKNOWLEDGMENTS}

We are grateful to Nicola Tomassia and Marco A Cassatella for technical assistance in ChIP analysis. We also thank to Maciej Tarkowski for critical comments. This work was supported by grants from the National Science Centre: 2014/13/B/NZ6/00235 to P.L., 2013/11/B/NZ6/02055 to H.C., and 2011/01/N/NZ6/01849 to M.C.

\section{DISCLOSURE}

The authors declared no conflict of interest.

(c) 2016 Society for Mucosal Immunology

\section{REFERENCES}

1. Mantovani, A., Cassatella, M.A., Costantini, C. \& Jaillon, S. Neutrophils in the activation and regulation of innate and adaptive immunity. Nat. Rev. Immunol. 11, 519-531 (2011).

2. Zhang, X., Majlessi, L., Deriaud, E., Leclerc, C. \& Lo-Man, R. Coactivation of Syk kinase and MyD88 adaptor protein pathways by bacteria promotes regulatory properties of neutrophils. Immunity 31, 761-771 (2009).

3. Tsuda, Y., Takahashi, H., Kobayashi, M., Hanafusa, T., Herndon, D.N. \& Suzuki, F. Three different neutrophil subsets exhibited in mice with different susceptibilities to infection by methicillin-resistant Staphylococcus aureus. Immunity 21, 215-226 (2004).

4. Bouabe, H., Liu, Y., Moser, M., Bösl, M.R. \& Heesemann, J. Novel highly sensitive IL-10-beta-lactamase reporter mouse reveals cells of the innate immune system as a substantial source of IL-10 in vivo. J. Immunol. 187, 3165-3176 (2011).

5. Ocucin, L. et al. Neutrophil IL-10 suppress peritoneal inflammatory monocytes during polymicrobial sepsis. J. Leukoc. Biol. 89, 423-432 (2011).

6. Greenblatt, M.B., Aliprantis, A., Hu, B. \& Glimcher, L.H. Calcineurin regulates innate antifungal immunity in neutrophils. J. Exp. Med. 207, 923-931 (2010).

7. Tosello Boari, J. et al. IL-17RA signaling reduces inflammation and mortality during Trypanosoma cruzi infection by recruiting suppressive IL-10producing neutrophils. PLoS Pathog. 8, e1002658 (2012).

8. De Santo, C. et al. Invariant NKT cells modulate the suppressive activity of IL-10-secreting neutrophils differentiated with serum amyloid A. Nat. Immunol. 11, 1039-1046 (2010).

9. De Santo, C., Salio, M., Dong, T., Reiter, Y. \& Cerundolo, V. Respond: "Failure to detect production of IL-10 by activated human neutrophils". Nat. Immunol. 12, 1018-1020 (2011).

10. Davey, M.S. et al. Failure to detect production of IL-10 by activated human neutrophils. Nat. Immunol. 12, 1017-1020 (2011).

11. Tamassia, N. et al. Cutting edge: an inactive chromatin configuration at the IL-10 locus in human neutrophils. J. Immunol. 190, 1921-1925 (2013).

12. Balderramas, H.A. et al. Human neutrophils produce IL-12, IL-10, PGE2 and LTB4 in response to Paracoccidioides brasiliensis involvement of TLR2, mannose receptor and dectin-1. Cytokine 67, 36-43 (2014).

13. Lewkowicz, P., Lewkowicz, N., Sasiak, A. \& Tchórzewski, H. Lipopolysaccharide-activated $\mathrm{CD} 4{ }^{+} \mathrm{CD} 25^{+} \mathrm{T}$ regulatory cells inhibit neutrophil function and promote their apoptosis and death. J. Immunol. 177, 7155-7163 (2006).

14. Lewkowicz, N., Klink, M., Mycko, M.P. \& Lewkowicz, P. Neutrophil $\mathrm{CD} 4{ }^{+} \mathrm{CD}_{25}{ }^{+}$Tregulatory cell interactions: a possible new mechanism of infectious tolerance. Immunobiology 218, 455-464 (2013).

15. Dima, A.A. et al. Comparison of segmentation algorithms for fluorescence microscopy images of cells. Cytometry A 79, 545-559 (2011).

16. Pillay, J. et al. A subset of neutrophils in human systemic inflammation inhibits T cell responses through Mac-1. J. Clin. Invest. 122, 327-336 (2012).

17. Himmel, M.E., Crome, S.Q., Ivison, S., Piccirillo, C., Steiner, T.S. \& Levings, M.K. Human CD4 ${ }^{+}$FOXP3 $^{+}$regulatory T cells produce CXCL8 and recruit neutrophils. Eur. J. Immunol. 41, 306-331 (2011).

18. Estaquier, J. \& Ameisen, J.C. A role for T-helper type-1 and type-2 cytokines in the regulation of human monocyte apoptosis. Blood 90, 1618-1625 (1997).

19. Turina, M., Hoth, J.J., Turpen, R.M., Scott, M.J. \& Cheadle, W.G. Alveolar interleukin-10 regulates neutrophil apoptosis in severely traumatized patients. J. Trauma 63, 733-739 (2007).

20. Schmidt, M., Lügering, N., Pauels, H.G., Schulze-Osthoff, K., Domschke, W. \& Kucharzik, T. IL-10 induces apoptosis in human monocytes involving 
the CD95 receptor/ligand pathway. Eur. J. Immunol. 30, 1769-1777 (2000).

21. Preshaw, P.M. \& Taylor, J.J. How has research into cytokine interactions and their role in driving immune responses impacted our understanding of periodontitis?. J Clin. Periodontol. 38 (Suppl 11), 60-84 (2011).

22. Zhang, H., Bennett, J.L. \& Verkman., A.S. Ex vivo spinal cord slice model of neuromyelitis optica reveals novel immunopathogenic mechanisms. Ann. Neurol. 70, 943-954 (2011).

23. Michael, B.D. et al. Post-acute serum eosinophil and neutrophilassociated cytokine/chemokine profile can distinguish between patients with neuromyelitis optica and multiple sclerosis; and identifies potential pathophysiological mechanisms - a pilot study. Cytokine 64, 90-96 (2013).

24. Munder, M. et al. Arginase I is constitutively expressed in human granulocytes and participates in fungicidal activity. Blood 105, 2549-2556 (2005).

25. Jacobsen, L.C., Theilgaard-Mönch, K., Christensen, E.I. \& Borregaard, N. Arginase 1 is expressed in myelocytes/metamyelocytes and localized in gelatinase granules of human neutrophils. Blood 109, 3084-3087 (2007).

26. Youn, J.I., Nagaraj, S., Collazo, M. \& Gabrilovich, D.I. Subsets of myeloidderived suppressor cells in tumor-bearing mice. J. Immunol. 181, 57915802 (2008).

27. Reglier, H., Arce-Vicioso, M., Fay, M., Gougerot-Pocidalo, M.A. \& CholletMartin, S. Lack of IL-10 and IL-13 production by human polymorphonuclear neutrophils. Cytokine 10, 192-198 (1988).

28. Smedman, C., Gårdlund, B., Nihlmark, K., Gille-Johnson, P., Andersson, J. \& Paulie, S. ELISpot analysis of LPS-stimulated leukocytes: human granulocytes selectively secrete IL-8, MIP-1beta and TNF-alpha. J. Immunol. Methods 346, 1-8 (2009).

29. Shimonkevitz, R.J., Bar-Or, D., Harris, L., Northrop, J. \& Yukl, R. Granulocytes, including neutrophils, synthesize $\mathrm{IL}-10$ after traumatic pancreatitis: case report. Trauma 48, 165-168 (2000).

30. Atallah, M. et al. Constitutive neutrophil apoptosis: regulation by cell concentration via S100 A8/9 and the MEK-ERK pathway. PLoS One 7, e29333 (2012).
31. Mayadas, T.N. \& Cullere, X. Neutrophil beta2 integrins: moderators of life or death decisions. Trends Immunol. 26, 388-395 (2005).

32. Keel, M. et al. Interleukin-10 counterregulates proinflammatory cytokineinduced inhibition of neutrophil apoptosis during severe sepsis. Blood 90 , 3356-3363 (1997).

33. Cox, G. IL-10 enhances resolution of pulmonary inflammation in vivo by promoting apoptosis of neutrophils. Am. J. Physiol. 271, 566-571 (1996).

34. Duffin, R., Leitch, A.E., Fox, S., Haslett, C. \& Rossi, A.G. Targeting granulocyte apoptosis: mechanisms, models, and therapies. Immunol. Rev. 236, 28-40 (2010).

35. Würstle, M.L., Laussmann, M.A. \& Rehm, M. The central role of initiator caspase-9 in apoptosis signal transduction and the regulation of its activation and activity on the apoptosome. Exp. Cell Res. 318, 1213-1220 (2012).

36. Bratton, S.B. \& Salvesen, G.S. Regulation of the Apaf-1-caspase-9 apoptosome. J. Cell Sci. 123, 3209-3214 (2010).

37. Gautam, S., Kirschnek, S., Wiesmeier, M., Vier, J. \& Häcker, G. Roscovitine-induced apoptosis in neutrophils and neutrophil progenitors is regulated by the Bcl-2-family members Bim, Puma, Noxa and Mcl-1. PLoS One 8, e79352 (2013).

38. Yamaguchi, T., Wing, J.B. \& Sakaguchi, S. Two modes of immune suppression by Foxp3 ${ }^{+}$regulatory T cells under inflammatory or noninflammatory conditions. Semin. Immunol. 23, 424-430 (2011).

39. Pattison, M.J., Mackenzie, K.F. \& Arthur, J.S. Inhibition of JAKs in macrophages increases lipopolysaccharide-induced cytokine production by blocking IL-10-mediated feedback. J. Immunol. 189, 2784-2792 (2012).

40. Wingerchuk, D.M., Hogancamp, W.F., O'Brien, P.C. \& Weinshenker, B.G. The clinical course of neuromyelitis optica (Devic's syndrome). Neurology 53, 1107-1114 (1999).

41. Barkhof, F. et al. Comparison of MRI criteria at first presentation to predict conversion to clinically definite multiple sclerosis. Brain 120, 2059-2069 (1997). 